\title{
LECTURES ON NEHARI'S THEOREM ON THE POLYDISK
}

\author{
MICHAEL T. LACEY \\ SCHOOL OF MATHEMATICS \\ GEORGIA INSTITUTE OF TECHNOLOGY \\ ATLANTA GA 30332
}

\begin{abstract}
We are concerned with Nehari's theorem on Hardy space on a polydisk. Define 'little' Hankel operators on product Hardy space $H^{2}\left(\mathbb{C}_{+}^{d}\right)$ by

$$
\mathrm{H}_{b} \varphi \stackrel{\text { def }}{=} \mathrm{P}_{\oplus} \mathrm{M}_{b} \bar{\varphi} \text {. }
$$

where $\mathrm{P}_{\oplus}$ is the orthogonal projection from $L^{2}\left(\mathbb{R}^{d}\right)$ to $H^{2}\left(\mathbb{C}_{+}^{d}\right)$ and $\mathrm{M}_{b}$ is the operator of multiplication by $b$. We present the proof of Ferguson and Lacey [27] and Lacey and Terwelleger [38] that we have the equivalence of norms

$$
\left\|H_{b}\right\| \cdot \simeq\|b\|_{B M O\left(\mathbb{C}_{+}^{d}\right)}
$$

for analytic functions $b$. Here, $B M O\left(\mathbb{C}_{+}^{d}\right)$ is the dual to $H^{1}\left(\mathbb{C}_{+}^{d}\right)$ as discovered by Chang and R. Fefferman. This article begins with the classical Nehari theorem, and presents the necessary background for the proof of the extension above. The proof of the extension is an induction on parameters, with a bootstrapping argument. Some of the more technical details of the earlier proofs are now seen as consequences of a paraproduct theory.
\end{abstract}

\section{Contents}

1. Introduction 2

2. Wavelets, $\mathrm{BMO}(\mathbb{R})$ and Paraproducts 4

2.1. Haar Wavelets 5

2.2. Meyer Wavelet 6

2.3. Hardy Space $H_{+}^{1}(\mathbb{T})$ and BMO $\quad 7$

2.4. Paraproducts $\quad 8$

3. The Nehari Theorem on the Disk 11

Research supported in part by a National Science Foundation Grant. The author is a Guggenheim Fellow. 
4. Aspects of Product Hardy Theory 17

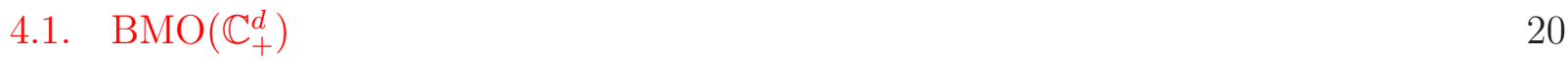

4.2. Journé's Lemma 20

5. Multiparameter Paraproducts 22

6. Nehari Theorem in Several Variables 25

7. Proof of Multiparameter Nehari Theorem 28

7.1. The Initial Lower Bound 28

7.2. The $\mathrm{BMO}\left(\mathbb{C}_{+}^{d}\right)$ lower bound. $\quad 29$

References $\quad 32$

\section{INTRODUCTION}

These notes concern the subject of Nehari's theorem, on Hardy space of the disk, and products of the disk. The theorem on the disk is classical, with three different approaches possible; the same question on products of the disk, the polydisk of the title, is a new result of the author, Sarah Ferguson and Erin Terwelleger $[27,38]$. The proof in the product setting is much more complicated, with currently only one proof known. It relies upon a delicate bootstrapping argument with an induction on parameters. These elements are suggested by the harmonic analysis associated with product theory, as developed by S.-Y. Chang, R. Fefferman and J.-L. Journé $[6-10,35,36]$. These notes will provide an approach to this result that is more leisurely than the research articles on the subject. We in particular include a great many references, and a description of related results and concepts. The proof of the main theorem we give is a little more 'structural' in that the main technical estimates are seen as consequences of a theory of paraproducts.

The key concepts of this paper concern the intertwined topics of Hankel operators, Hardy space, Hilbert transforms, commutators, and paraproducts. Let us describe Hankel matrices. 
Consider a function $b$ on $L^{2}(\mathbb{T})$, and the operator $\mathrm{M}_{b}$ of pointwise multiplication by $b$. That is, $\mathrm{M}_{b} \varphi \stackrel{\text { def }}{=} b \cdot \varphi$. Now, $L^{2}(\mathbb{T})$ has the exponential basis. We view the circle as embedded in the natural way in the complex plane, so that a relevant basis is $\left\{z^{n}: n \in \mathbb{Z}\right\}$. The decomposition of functions in this basis of course generates the Fourier transform:

$$
\widehat{f}(n) \stackrel{\text { def }}{=} \int_{\mathbb{T}} f(z) z^{-n}|d z|
$$

In the exponential basis, $\mathrm{M}_{b}$ has a matrix form,

$$
\mathrm{M}_{b} \longleftrightarrow\{\widehat{b}(i-j): i, j \in \mathbb{Z}\}
$$

Restrictions of this matrix give Hankel and Toeplitz operators.

The restriction of the matrix to the upper quadrant $\mathbb{N} \times \mathbb{N}$ is a Toeplitz matrix. Namely, $\mathrm{T}=\left\{t_{i j}: i, j \in \mathbb{N}\right\}$ is a Toeplitz matrix iff $t_{i j}=\alpha_{i-j}$ for some numerical sequence $\alpha$ on $\mathbb{Z}$. In this note, we are principally interested in the boundedness properties of operators. It is easy to see that Toeplitz matrix is bounded iff the sequence $\alpha_{j}$ are the Fourier coefficients of a bounded function. How this statement changes for Hankel matrices occupies our attention.

Restricting the matrix to say the quadrant $\mathbb{N} \times(-\mathbb{N})$ gives a Hankel matrix. Namely, $\mathrm{H}=\left\{h_{i j}: i, j \in \mathbb{N}\right\}$ is a Hankel matrix iff $h_{i j}=\alpha_{i+j}$ for some numerical sequence on $\mathbb{N}$.

In passing to these restrictions of the matrix for $\mathrm{M}_{b}$, we are implicitly restricting the matrix on $\ell^{2}(\mathbb{Z})$ to one on $\ell^{2}(\mathbb{N})$. Namely, a Hankel and Toeplitz matrix are operators on $\ell^{2}(\mathbb{N})$. The natural analog in $L^{2}(\mathbb{T})$ is Hardy space $H^{2}(\mathbb{T})$. By definition, $H^{2}(\mathbb{T})=H_{+}^{2}(\mathbb{T})$ is the closed subspace of $L^{2}(\mathbb{T})$ generated by $\left\{z^{n}: n \geq 0\right\}$. It is natural to call these functions analytic, as $f \in H^{2}(\mathbb{T})$ admit an analytic extension to the disk $\mathbb{D}$ given by

$$
F(z)=\sum_{n \geq 0} \widehat{f}(n) z^{n} .
$$

Functions in $H_{-}^{2}(\mathbb{T})=L^{2}(\mathbb{T}) \ominus H^{2}(\mathbb{T})$ are referred to as antianalytic.

Let us describe the Hankel operators on $H^{2}(\mathbb{T})$. Let $\mathrm{P}_{ \pm}$be the orthogonal projection from $L^{2}(\mathbb{T})$ onto the subspace $H_{ \pm}^{2}(\mathbb{T})$. A Hankel operator with symbol $b$ is an operator $\mathrm{H}_{b}$ from $H_{+}^{2}(\mathbb{T})$ to $H_{-}^{2}(\mathbb{T})$ given by $\mathrm{H}_{b} \varphi \stackrel{\text { def }}{=} \mathrm{P}_{-} \mathrm{M}_{b} \bar{\varphi}$. It is clear that this definition only depends on the analytic part of $b$.

1.1. Remark. The placement of the conjugate symbol is somewhat arbitrary, and is adopted in this way only for convenience. Richard Rochberg avoids such complications by defining Hankel operators as bilinear operators B from $H^{2} \times H^{2}$ into $\mathbb{C}$, which are linear on products: $\mathrm{B}(\varphi, \psi)=\mathrm{L}(\varphi \cdot \psi)$ for a linear functional $\mathrm{L}$. 
A central operator on $\ell^{2}(\mathbb{N})$ the shift operator $S\left(\alpha_{0}, \alpha_{1}, \ldots\right) \stackrel{\text { def }}{=}\left(0, \alpha_{0}, \alpha_{1}, \ldots\right)$. Hankel operators $\mathrm{H}$ are distinguished by their intertwining with the shift operator:

$$
\mathrm{HS}=\mathrm{S}^{*} \mathrm{H} \text {. }
$$

Proof is left for the reader.

The shift operator on Hardy space $H^{2}(\mathbb{D})$ is given by multiplication by $z$, and Hankel operators on Hardy space enjoy the same intertwining with the shift operator.

While we have taken pains to outline these initial observations on the integers and the circle, there is an equivalent formulation on the real line. To be specific, on $L^{2}(\mathbb{R})$, we have the Fourier transform

$$
\widehat{f}(\xi)=\int f(x) \mathrm{e}^{-i \xi x} d x
$$

Define the orthogonal projections onto positive and negative frequencies

$$
\mathrm{P}_{ \pm} f(x) \stackrel{\text { def }}{=} \int_{\mathbb{R}_{ \pm}} \widehat{f}(\xi) \mathrm{e}^{i \xi x} d x .
$$

Define Hardy spaces $H_{ \pm}^{2}(\mathbb{R}) \stackrel{\text { def }}{=} \mathrm{P}_{ \pm} L^{2}(\mathbb{R})$. Functions $f \in H_{+}^{2}(\mathbb{R})$ admit an analytic extension to the upper half plane $\mathbb{C}_{+}$. As in the case of the disk, it is convenient to refer to functions in $H_{+}^{2}(\mathbb{R})$ as analytic.

A Hankel operator with symbol $b$ is then a linear operator from $H_{+}^{2}(\mathbb{R})$ to $H_{-}^{2}(\mathbb{R})$ given by $\mathrm{H}_{b} \varphi \stackrel{\text { def }}{=} \mathrm{P}_{-} \mathrm{M}_{b} \bar{\varphi}$. This only depends on the analytic part of $b$.

It will be convenient to consider some of our proofs in the setting of the real line. For, while it is equivalent to work on any of the three settings, the real line has a natural dilation structure which simplifies certain aspects of the argument.

Acknowledgment. These notes were prepared while in residence at the University of British Columbia, for a conference "Harmonic Analysis at Sapporo, Japan" held in August 2005.

\section{Wavelets, BMO $(\mathbb{R})$ and Paraproducts}

Ultimately, we are interested in characterizations of BMO. This class of functions have delicate properties, sensitive to locations in both time and frequency. Wavelets turn out to be very useful in analyzing their behavior. We recall some basic facts about two distinct classes of wavelets, namely the Haar and Meyer wavelets.

Throughout this paper, $\mathcal{D}$ denotes the dyadic grid. Thus,

$$
\mathcal{D} \stackrel{\text { def }}{=}\left\{\left[j 2^{k},(j+1) 2^{k}\right): j, k \in \mathbb{Z}\right\} \text {. }
$$


Define translation and dilation operators by

$$
\begin{aligned}
\operatorname{Tr}_{y} f(x) & \stackrel{\text { def }}{=} f(x-y), \quad y \in \mathbb{R}, \\
\operatorname{Dil}_{s}^{p} f(x) & \stackrel{\text { def }}{=} s^{-1 / p} f(x / s), \quad 0<s, p<\infty, \\
\operatorname{Dil}_{I}^{p} f(x) & \stackrel{\text { def }}{=} \operatorname{Tr}_{c(I)} \operatorname{Dil}_{|I|}^{p} f(x), \quad I \text { is an interval. }
\end{aligned}
$$

In the second definition, $s$ denotes the scale of the dilation, and the normalization is chosen to preserve $L^{p}(\mathbb{R})$ norm. In the last definition, we extend the definition of dilation to an interval, which incorporates a translation to the center of $I$, denoted $c(I)$, and a dilation by the scale of $I$.

2.1. Haar Wavelets. The notations $h=h^{0}$ and $h^{1}$ are reserved for the functions

$$
h=h^{0}=-\mathbf{1}_{[0,1 / 2)}+\mathbf{1}_{[1 / 2,1]}, \quad h^{1}=\mathbf{1}_{[0,1]} .
$$

Here, the superscript ${ }^{0}$ means that that the function has mean zero, while the superscript ${ }^{1}$ means that the function does not have mean zero. We will use these two definitions in our discussion of paraproducts; the function $h^{0}$ is used most of the time, and we will frequently suppress the superscript when using these functions.

For any interval $I$, we can define $h_{I}^{\epsilon} \stackrel{\text { def }}{=} \operatorname{Dil}_{I}^{2} h^{\epsilon}$. The Haar wavelets are then given by $\left\{h_{I}^{0}: I \in \mathcal{D}\right\}$. These functions are an orthogonal basis on $L^{2}(\mathbb{R})$, which extend to an unconditional basis on $L^{p}(\mathbb{R})$ for $1<p<\infty$. In particular, the Littlewood Paley inequalities become

2.5. Theorem. We have the estimates

$$
\|f\|_{p} \simeq\left\|\left[\sum_{I \in \mathcal{D}} \frac{\left|\left\langle f, h_{I}\right\rangle\right|^{2}}{|I|} \mathbf{1}_{I}\right]^{1 / 2}\right\|_{p}, \quad 1<p<\infty .
$$

More generally, if $\varphi$ is adapted to $[0,1]$ and has mean zero, we have the square function below maps $L^{p}$ into itself for all $1<p<\infty$.

$$
\mathrm{S} f \stackrel{\text { def }}{=}\left[\sum_{I \in \mathcal{D}} \frac{\left|\left\langle f, \varphi_{I}\right\rangle\right|^{2}}{|I|} \mathbf{1}_{I}\right]^{1 / 2} \text {. }
$$

One natural extension of these estimates to the cases of $p=1$ and $p=\infty$ can be taken as the definitions of Hardy space $H^{1}$ and BMO.

We should also mention that

$$
\mathrm{M}_{\mathrm{dy}} f(x)=\sup _{I \in \mathcal{D}} \frac{\left|\left\langle f, h_{I}\right\rangle\right|}{\sqrt{|I|}} \mathbf{1}_{I}
$$

is the dyadic maximal function. It maps $L^{p}$ into itself for all $1<p \leq \infty$. The proof of this theorem can appeal to probabilistic methods. Indeed, the expansion of a function in 
a Haar basis is a martingale. This fact lies behind the very successful application of Haar functions to establish a range of deeper properties of singular integrals, including the Hilbert transform. These properties include the UMD theory of Burkholder [3] and Bourgain [2] ; matrix valued paraproducts, as discussed in articles by a range of authors $[46,47,51,58,59]$ and the Nazarov Treil Volberg extension of the Calderón Zygmund theory [49,50], as well as their discussion of the Bellman function approach [48,52].

More generally, the definition of the maximal function is

$$
\mathrm{M} f(x) \stackrel{\text { def }}{=} \sup _{t>0}(2 t)^{-1} \int_{-t}^{t} f(x-y) d y
$$

where it is essential that we do not impose absolute values inside the integral. Define the Hilbert transform

$$
\mathrm{H} f(x) \stackrel{\text { def }}{=}-\mathrm{P}_{-}+\mathrm{P}_{+} f(x)=\mathrm{p} \cdot \mathrm{v} \cdot \frac{1}{\pi} \int f(x-y) \frac{d y}{y} .
$$

2.2. Meyer Wavelet. Y. Meyer [42] found a Schwartz function $w$, with

$$
\widehat{w} \text { is supported on } 2 \pi \leq|\xi| \leq 8 \pi \text {, }
$$

and the functions $\left\{w_{I}: I \in \mathcal{D}\right\}$ form an orthonormal basis for $L^{2}(\mathbb{R})$. Here, we use the same notation as in the case of the Haar basis, $w_{I}=\operatorname{Dil}_{I}^{2}$.

As with the Haar basis, these extend to an unconditional basis on $L^{p}(\mathbb{R})$, for $1<p<\infty$. As concerns the Hilbert transform, observe that

$$
\mathrm{H} f=\sum_{I \in \mathcal{D}}\left\langle f, w_{I}\right\rangle \mathrm{H} w_{I},
$$

and that the functions $\mathrm{H} w_{I}=\operatorname{Dil}_{I}^{2}(\mathrm{H} w)$ have the same decay and Fourier localization properties of the Meyer wavelet.

Similarly, if $f \in H^{2}(\mathbb{R})$, we have

$$
\begin{aligned}
f=\mathrm{P}_{+} f & =\sum_{I \in \mathcal{D}}\left\langle f, w_{I}\right\rangle w_{I} \\
& =\mathrm{P}_{+}\left[\sum_{I \in \mathcal{D}}\left\langle\mathrm{P}_{+} f, w_{I}\right\rangle w_{I}\right] \\
& =\sum_{I \in \mathcal{D}}\left\langle\mathrm{P}_{+} f, w_{I}\right\rangle \mathrm{P}_{+} w_{I}
\end{aligned}
$$

Therefore, $\left\{\mathrm{P}_{+} w_{I}: I \in \mathcal{D}\right\}$ is a basis for $H^{2}(\mathbb{R})$. 
2.3. Hardy Space $H_{+}^{1}(\mathbb{T})$ and BMO. For $1 \leq p \leq \infty$, the Hardy space $H^{p}(\mathbb{T})$ is the closure in $L^{p}(\mathbb{T})$ norm of the span of the exponentials $\left\{z^{n}: n \in \mathbb{N}\right\}$. A function $f \in H^{p}(\mathbb{T})$ has an analytic extension $F$ to the disk. Indeed, this extension is

$$
F(z)=\sum_{n=0}^{\infty} \widehat{f}(n) z^{n} .
$$

and the $H^{p}(\mathbb{T})$ norm can be taken to be

$$
\|f\|_{H^{p}(\mathbb{T})} \stackrel{\text { def }}{=} \sup _{0<r<1}\|F(r z)\|_{L^{p}(\mathbb{T})}
$$

Concerning the space $H^{1}(\mathbb{T})$, the following classical property is central to us.

2.11. Proposition. Each function $f \in H^{1}(\mathbb{T})$ is a product of functions $f_{1}, f_{2} \in H^{2}(\mathbb{T})$, in particular, $f_{1}$ and $f_{2}$ can be chosen so that

$$
\|f\|_{H^{1}}=\left\|f_{1}\right\|_{H^{2}}\left\|f_{2}\right\|_{H^{2}}
$$

2.12. Remark. In the product setting to which we turn to next, this last property fails. Indeed, part of the interest of our results is that while simple factorization will fail, a notion of weak factorization is in fact true.

2.13. Remark. The investigation of the failure of factorization is an intricate one. In the product setting, Rudin [64] proved the failure of factorization in the case of $\mathbb{D}^{d}$ for $d \geq 4$; Miles [43] improved the result to $d \geq 3$; Rosay [63] in the case of $d=2$ showed that the set of functions in $H^{1}(\mathbb{D} \otimes \mathbb{D})$ which factor is of the first category. This problem is also of interest in the Bergman space setting. See [29,31,32]. See [29] for information about this question in other spaces of analytic functions.

We are especially interested in the Hardy space $H^{1}(\mathbb{R})$. It is technically easier to discuss the real Hardy space $\operatorname{Re}\left(H^{1}\right)$ consisting of the real part of functions in $H^{1}$.

2.14. Theorem. We have the equivalence of norms

$$
\|f\|_{\operatorname{Re}\left(H^{1}\right)} \simeq\|f\|_{1}+\|\mathrm{H} f\|_{1} \simeq\|\mathrm{S} f\|_{1} \simeq\|\mathrm{M} f\|_{1}
$$

Here $\mathrm{S}$ is as in (2.6).

Any standard reference in the subject will include a proof of this result. Historically, this kind of characterization was an essential precursor to the proof of Fefferman and Stein of $H^{1}$ and BMO duality.

Indeed, one can use this characterization of $H^{1}$ in terms of atoms (which we don't define here) which lead immediately to 
2.15. Theorem $\left(H^{1}(\mathbb{R})\right.$ - $\mathrm{BMO}(\mathbb{R})$ duality). The dual of $\operatorname{Re}\left(H^{1}(\mathbb{R})\right)$ is $\mathrm{BMO}(\mathbb{R})$ with norm

$$
\begin{aligned}
\|f\|_{\mathrm{BMO}(\mathbb{R})} & \stackrel{\text { def }}{=} \sup _{J \text { is an interval }}\left[|J|^{-1} \sum_{\substack{I \in \mathcal{D} \\
I \subset J}}\left|\left\langle f, w_{R}\right\rangle\right|^{2}\right] \\
& \simeq \sup _{J \text { is an interval }}\left[|J|^{-1} \int_{J}\left|f(x)-\mu_{J}\right|^{2} d x\right]^{1 / 2}
\end{aligned}
$$

where $\mu_{J}=|J|^{-1} \int_{J} f(y) d y$.

The second definition has the advantage of being intrinsic to $f$, but has the disadvantage of not having a suitable generalization to higher parameters. Thus, we have stressed the first definition in terms of wavelets. There is nothing special about the Meyer wavelet appearing here. It can be replaced by any wavelet, including the Haar wavelet in this context.

One nice feature of the Meyer wavelet, is that if we replace the functions $w_{R}$ by their analytic projections, we obtain a completely analogous definition of analytic BMO, the dual to $H^{1}(\mathbb{T})$. .

2.4. Paraproducts. Paraproduct is the term used to refer to any of a wide variety of objects that are a variant of a product of two functions. A Hankel operator is one such example, but our purpose in this section is to describe a class of more naive examples.

Consider the operation $\mathrm{M}_{b} f$, where we take both $b$ and $f$ to have finite Haar expansion on the real line.

$$
\mathrm{M}_{b} f=\sum_{I, J \in \mathcal{D}}\left\langle b, h_{I}\right\rangle\left\langle f, h_{J}\right\rangle h_{I} \cdot h_{J}
$$

Restrict the sum above to $I \subsetneq J$, and observe that

$$
\begin{aligned}
\operatorname{Para}_{\text {Haar }}(b, f) & =\sum_{\substack{I, J \in \mathcal{D} \\
I \subsetneq J}}\left\langle b, h_{I}\right\rangle\left\langle f, h_{J}\right\rangle h_{I} \cdot h_{J} \\
& =\sum_{I \in \mathcal{D}} \frac{\left\langle b, h_{I}\right\rangle}{\sqrt{|I|}}\left\langle f, h_{I}^{1}\right\rangle h_{I}
\end{aligned}
$$

Here, we are appealing to this property of Haar functions:

$$
\left\langle h_{J}, h_{I}^{1}\right\rangle= \begin{cases}0 & I \cap J=\emptyset, \quad J \subset I, \\ \frac{h_{J}(c(I))}{\sqrt{|I|}} & I \subsetneq J .\end{cases}
$$

The point of this is that while the operator norm of $\mathrm{M}_{b}$ is $\|b\|_{\infty}$, while the norm of the operator in $(2.16)$ is in general somewhat smaller. Para $\operatorname{Haar}(b, \cdot)$ is a paraproduct. 
Paraproducts admit a more general definition, which is the point of this definition. For an interval $I$, we say that $\varphi$ is adapted to $I$ iff $\|\varphi\|_{2}=1$ and

$$
\left|\mathrm{D}^{n} \varphi(x)\right| \lesssim|I|^{-n-\frac{1}{2}}\left(1+\frac{|x-c(I)|}{|I|}\right)^{-N}, \quad n=0,1
$$

Here, $c(I)$ denotes the center of $I$, and $N$ is a large integer, whose exact value need not concern us $\mathrm{D}$ denotes the derivative operator. We shall consistently work with functions which have $L^{2}$ norm at most one. Some of these functions we will also insist to have integral zero.

We take $\left\{\varphi_{I}^{\varepsilon}: I \in \mathcal{D}\right\}, \varepsilon \in\{0,1\}$ to be functions adapted to $I \in \mathcal{D}$. The functions $\varphi_{I}^{0}$ are of mean zero. Then, set

$$
\operatorname{Para}(b, f) \stackrel{\text { def }}{=} \sum_{I \in \mathcal{D}} \frac{\left\langle b, \varphi_{I}^{0}\right\rangle}{\sqrt{|I|}}\left\langle f, \varphi_{I}^{1}\right\rangle \varphi_{I}^{0}
$$

2.18. Theorem. For $1<p<\infty$, we have the inequality

$$
\left\|\operatorname{Para}_{\mathrm{Haar}}(b, \cdot)\right\|_{p \rightarrow p} \lesssim\|b\|_{\mathrm{BMO}_{\mathrm{dy}}},
$$

where the last norm is dyadic BMO norm defined by

$$
\|b\|_{\mathrm{BMO}_{\mathrm{dy}}} \stackrel{\text { def }}{=} \sup _{I \in \mathcal{D}}\left[|I|^{-1} \sum_{J \subset I}\left|\left\langle b, h_{I}\right\rangle\right|^{2}\right]^{1 / 2} .
$$

For the operators Para, we have

$$
\|\operatorname{Para}(b, \cdot)\|_{p \rightarrow p} \lesssim\|b\|_{\mathrm{BMO}}
$$

It is essential in this formulation that we do not let the function $h_{I}^{1}$, which does not have mean zero, fall on the BMO function $b$. This point of view is very helpful in obtaining upper bounds for other commutators, Hankel operators and related objects.

It is useful to observe that paraproducts can arise in a wide variety of forms, in particular, the classical approach of Coffman and Meyer relies upon the ' $\mathrm{P}_{t}-\mathrm{Q}_{t}$ ' formalism. A form useful to us is as follows. For the Meyer wavelet $w$, with it's antianalytic and analytic parts, respectively $u$ and $v$, let us set

$$
\begin{gathered}
\Delta \mathrm{U}_{j} \stackrel{\text { def }}{=} \sum_{\substack{I \in \mathcal{D} \\
|I|=2^{j}}} u_{I} \otimes u_{I} \\
\mathrm{U}_{j} \stackrel{\text { def }}{=} \sum_{k \geq j} \Delta \mathrm{U}_{k}
\end{gathered}
$$

The following Theorem concerns a paraproduct which is quite close to being a Hankel operator; it's bounds are a corollary to the previous Theorem. 
2.23. Theorem. We have the estimate

$$
\left\|\sum_{j \in \mathbb{Z}}\left(\Delta \mathrm{U}_{j} b\right) \cdot \overline{\left(\mathrm{U}_{j}\right) \varphi}\right\|_{2} \lesssim\|b\|_{\mathrm{BMO}(\mathbb{R})}\|\varphi\|_{2}
$$

Proof. Let us first consider terms like

$$
\sum_{j \in \mathbb{Z}} \Delta \mathrm{U}_{j} \cdot \overline{\Delta \mathrm{U}_{j+k}}, \quad 0 \leq k \leq 8 .
$$

We take e.g. $|k| \leq 8$ due to the compact frequency support of the Meyer wavelets, as will be come clear momentarily. The assertion is that each of these is a bounded operator, provided $b \in \operatorname{BMO}(\mathbb{R})$.

We control this expression in a brute force method, which we will appeal to twice. Fix $k$ and a map $\pi: \mathcal{D} \longrightarrow \mathcal{D}$ so that $|\pi(I)|=2^{k} I$ and $(A-1)|I| \leq \operatorname{dist}(I, J)<A|I|$, for some fixed integer $A$.

Write

$$
\psi_{I} \stackrel{\text { def }}{=} \sqrt{|I|} u_{I} \cdot \overline{u_{\pi(I)}}
$$

Observe that $A^{100} \psi_{I}$ is adapted to $I$ with constant independent of $A$ (and we certainly do not assert that it has integral zero!). This is the only observation we need to make to conclude that

$$
\left\|\sum_{I \in \mathcal{D}} \frac{\left\langle b, u_{I}\right\rangle}{\sqrt{|I|}} \overline{\left\langle\varphi, u_{\pi(I)}\right\rangle} \psi_{I}\right\|_{2} \lesssim A^{-100}\|b\|_{\mathrm{BMO}(\mathbb{R})}\|\varphi\|_{2} .
$$

This is summed over the different values of $A$ and $\pi$ to conclude the estimate

$$
\left\|\sum_{j \in \mathbb{Z}} \Delta \mathrm{U}_{j} b \cdot \overline{\Delta \mathrm{U}_{j+k} \varphi},\right\|_{2} \lesssim\|b\|_{\mathrm{BMO}(\mathbb{R})}\|\varphi\|_{2}, \quad 0<k<8 .
$$

Associated with the Meyer wavelet is a 'father wavelet,' a function $W$ of non zero mean, for which $w=W-\operatorname{Dil}_{1 / 2}^{1} W$. Using this, we see that

$$
\mathrm{U}_{j} f=\sum_{|I|=2^{j}}\left\langle f, W_{I}\right\rangle W_{I} .
$$

We then have

$$
\sum_{j \in \mathbb{Z}}\left(\Delta \mathrm{U}_{j} b\right) \cdot \overline{\mathrm{U}_{j+9} \varphi}=\sum_{\substack{I, J \in \mathcal{D} \\ 2^{9}|I| \leq|J|}} \frac{\left\langle b, u_{I}\right\rangle}{\sqrt{|I|}} \overline{\left\langle\mathrm{P}_{+} \varphi, W_{J}\right\rangle} \sqrt{I} u_{I} \cdot W_{J} .
$$

Observe that in contrast to the previous case, we are taking inner products $\left\langle\mathrm{P}_{+} f, W_{J}\right\rangle$ and $W_{J}$ will have a non zero mean. 
Nevertheless, this last sum can be controlled by the brute force method used above, with this observation. Take two dyadic intervals $I$ and $J$ with $2^{9}|I|=|J|$ and $(A-1)|I| \leq$ $\operatorname{dist}(I, J) \leq A|I|$, for integer $A$. Then,

$$
A^{100} \sqrt{I} u_{I} \cdot W_{J}
$$

is adapted to $I$ with constant independent of $A$, and has integral zero. The reason for this stems from (2.10). The Fourier transform of the product is supported in the convolution of the supports of the Fourier transforms of the two functions. Since $I$ has the smaller scale, the Fourier transform of $u_{I}$ is supported a very great distance from the origin, hence the Fourier transform is zero at the origin. This completes the proof.

\section{The Nehari Theorem on the Disk}

The classical result that we are interested in is:

Nehari Theorem ([53]). The Hankel operator $\mathrm{H}_{b}$ from $H_{+}^{2}(\mathbb{T})$ to $H_{+}^{2}(\mathbb{T})$ iff there is a bounded function $\beta$ with $P_{+} b=P_{+} \beta$. Moreover,

$$
\left\|\mathrm{H}_{b}\right\|=\inf _{\beta: \mathrm{P}_{+}=\mathrm{P}_{+} b}\|\beta\|_{\infty}
$$

There are three proofs of this fact in the literature. In the new results, we will need to rely upon methods from two of these methods.

Factorization. Given a bounded Hankel operator $\mathrm{H}_{b}$, we want to show that we can construct a bounded function $\beta$ so that the analytic part of $b$ and $\beta$ agree.

This proof is the one found by Nehari [53]. We begin with a basic computation of the norm of the Hankel operator $\mathrm{H}_{b}$ :

$$
\begin{aligned}
& \left\|\mathrm{H}_{b}\right\|=\sup _{\|\varphi\|_{H_{+}^{2}(\mathbb{T})}=1} \sup _{\|\psi\|_{H_{+}^{2}(\mathbb{T})}=1} \int \mathrm{H}_{b} \psi \cdot \bar{\varphi} d x \\
& =\sup _{\|\varphi\|_{H_{+}^{2}(\mathbb{T})}=1\|\psi\|_{H_{+}^{2}(\mathbb{T})}=1} \int \mathrm{P}_{+} \mathrm{M}_{b} \bar{\psi} \cdot \bar{\varphi} d x \\
& =\sup _{\|\varphi\|_{H_{+}^{2}(\mathbb{T})}=1\|\psi\|_{H_{+}^{2}(\mathbb{T})}=1} \int\left(\mathrm{P}_{+} b\right) \overline{\psi \cdot \varphi} d x \\
& =\sup _{\|\varphi\|_{H_{+}^{2}(\mathbb{T})}=1} \sup _{\|\psi\|_{H_{+}^{2}(\mathbb{T})}=1}\left\langle\left(\mathrm{P}_{+} b\right), \psi \cdot \varphi\right\rangle
\end{aligned}
$$

But, the $H^{1}(\mathbb{T})=H^{2}(\mathbb{T}) \cdot H^{2}(\mathbb{T})$, as we recalled in Proposition 2.11. We read from the equality above that the analytic part of $b$ defines a bounded linear functional on $H^{1}(\mathbb{T})$ a subspace of $L^{1}(\mathbb{T})$. 
The Hahn Banach Theorem applies, giving us an extension of this linear functional to all of $L^{1}$, with the same norm. But a linear function on $L^{1}$ is a bounded function, hence we have constructed a bounded function $\beta$ with the same analytic part as $b$.

Duality. In this proof, the $H^{1}$ - BMO duality is decisive. The calculation (3.1) shows that $\mathrm{P}_{+} b$ is a bounded linear functional on $H^{1}$. Therefore, we have

$$
\left\|\mathrm{H}_{b}\right\| \simeq\left\|\mathrm{P}_{+} b\right\|_{\mathrm{BMO}}
$$

(This is not equality, since we are not choosing to adopt a canonical norm for BMO.) In addition, we have $\mathrm{BMO}=L^{\infty}+\mathrm{H} L^{\infty}$, where $\mathrm{H}$ is the Hilbert transform. Therefore, we can select $\beta \in L^{\infty}$ which has the same analytic part as $b$.

3.2. Remark. Historically, this proof came last; it depends critically upon the Fefferman Stein $H^{1}$-BMO duality, which was not established until the 1970 's, see [22].

The AAK Method. Adamjan, Arov and Krein [1] invented a method based upon an dilation ${ }^{1}$ property of operators on Hilbert space. This method avoids the finer aspects of Hardy spaces.

We are given a Hankel matrix which is bounded from $\ell^{2}(\mathbb{N})$ to itself, and we seek to extend it to a bounded matrix on $\ell^{2}(\mathbb{Z})$, with the same norm. This is an inductive process, with the first step being that we seek to add, say, a row to the 'top' of the matrix:

$$
\left[\begin{array}{cccc}
a_{0} & a_{1} & a_{2} & \ldots \\
a_{1} & a_{2} & a_{3} & \ldots \\
a_{2} & a_{3} & a_{4} & \ldots \\
\vdots & \vdots & \vdots & \ddots
\end{array}\right] \longrightarrow\left[\begin{array}{cccc}
* & a_{0} & a_{1} & \ldots \\
a_{0} & a_{1} & a_{2} & \ldots \\
a_{1} & a_{2} & a_{3} & \ldots \\
a_{2} & a_{3} & a_{4} & \ldots \\
\vdots & \vdots & \vdots & \ddots
\end{array}\right]
$$

Namely, we seek to choose a value of $*$ to put on the upper left hand coordinate so that the two matrices have the same norm.

This in fact can be done, and leads to the following Proposition.

3.3. Proposition. Consider two Hilbert spaces $\mathcal{G}$ and $\mathcal{H}$, and consider linear operators from $\mathcal{G} \oplus \mathcal{H}$ into itself of the form

$$
\mathrm{U}=\left[\begin{array}{ll}
\mathrm{X} & \mathrm{C} \\
\mathrm{A} & \mathrm{B}
\end{array}\right]
$$

where $\mathrm{X}: \mathcal{G} \rightarrow \mathcal{G} ; \mathrm{A}: \mathcal{G} \rightarrow \mathcal{H} ; \mathrm{B}: \mathcal{H} \rightarrow \mathcal{G}$; and $\mathrm{C}: \mathcal{H} \rightarrow \mathcal{G}$. We presume that $\mathrm{A}, \mathrm{B}, \mathrm{C}$ are prescribed in advance. Then we can select $\mathrm{X}$ so that

$$
\|\mathrm{U}\|=\max \{\|A\|,\|B\|,\|C\|\} .
$$

\footnotetext{
${ }^{1}$ This dilation property is distinct from the dilation property of the real line used in other parts of this paper.
} 
Given a Hankel matrix $\mathrm{H}=\left\{a_{j+k}: j, k \in \mathbb{N}\right\}$, we apply the proposition above with

$$
\mathrm{A}=\left[\begin{array}{c}
a_{0} \\
a_{1} \\
a_{2} \\
\vdots
\end{array}\right], \quad \mathrm{B}=\left[\begin{array}{cccc}
a_{1} & a_{2} & a_{3} & \ldots \\
a_{2} & a_{3} & \ldots & \\
\vdots & \vdots & \ddots &
\end{array}\right], \quad \mathrm{C}=\left[\begin{array}{llll}
a_{0} & a_{1} & a_{2} & \ldots
\end{array}\right]
$$

By the proposition, we can choose $a_{-1}$ so that the norm of $\mathrm{H}$ is the norm of

$$
\left[\begin{array}{cccc}
a_{-1} & a_{0} & a_{1} & \cdots \\
a_{0} & a_{1} & a_{2} & \cdots \\
a_{1} & a_{2} & a_{3} & \cdots \\
\vdots & \vdots & \vdots & \ddots
\end{array}\right]
$$

By induction, we can extend the Hankel operator to $\ell^{2}(\mathbb{Z})$ to itself, as a bounded operator with the same norm. The conclusion of Nehari's theorem then follows.

3.4. Remark. This method has found many deep extensions to Hankel matrices whose entries are themselves operators. We refer the reader to Nikolski [54], as well as Nikolski [55] and Peller [57] for very interesting discussions of the method of Adamyan, Arov and Krein. This is relevant for us, as in the extension to the product setting, we consider Hankel matrices whose entries are also Hankelian. Cotlar and Sadosky have studied extensions of this method to the polydisk in a sequence of papers $[13-16,18,19]$

3.1. Commutators. Commutators are very useful in measuring, in a quantitative way, the distance from being abelian. They are relevant for us as the Nehari theorem has an equivalent formulation in terms of commutators of multiplication operators and the Hilbert transform.

3.5. Theorem. We have the equivalence

$$
\left\|\left[\mathrm{M}_{b}, \mathrm{H}\right]\right\|_{2 \rightarrow 2} \simeq\|b\|_{\mathrm{BMO}}
$$

Here, $\mathrm{BMO}=\left(\operatorname{Re} H^{1}(\mathbb{T})\right)^{*}$ is real $\mathrm{BMO}$.

The proof in this circumstance is immediate: Observe that $\mathrm{P}_{\mp}\left[\mathrm{M}_{b}, \mathrm{H}\right] P_{ \pm}$is itself a Hankel operator, or a conjugate of a Hankel operator. Specifically,

$$
\begin{array}{ll}
\mathrm{P}_{+}\left[\mathrm{M}_{b}, \mathrm{H}\right] \mathrm{P}_{+}=0, & \mathrm{P}_{-}\left[\mathrm{M}_{b}, \mathrm{H}\right] \mathrm{P}_{-}=0 \\
\mathrm{P}_{+}\left[\mathrm{M}_{b}, \mathrm{H}\right] \mathrm{P}_{-}=-\mathrm{P}_{+} \mathrm{M}_{b} \mathrm{P}_{-}, & \mathrm{P}_{-}\left[\mathrm{M}_{b}, \mathrm{H}\right] \mathrm{P}_{+}=\mathrm{P}_{-} \mathrm{M}_{b} \mathrm{P}_{+}
\end{array}
$$

and the last two operators are orthogonal, and Hankel operators.

The Theorem admits many extensions. For instance, we continue to have the equivalence

$$
\left\|\left[\mathrm{M}_{b}, \mathrm{H}\right]\right\|_{p \rightarrow p} \simeq\|b\|_{\mathrm{BMO}}, \quad 1<p<\infty .
$$


Indeed, assuming the commutator with symbol $b$ is bounded on $L^{p}$, the same is true on the dual index $L^{p^{\prime}}$. But then, by interpolation, the commutator is bounded on $L^{2}$, and we can appeal to the Theorem above to deduce that the symbol is in BMO.

The upper bound on the $L^{p}$ norm of the commutator follows by considering the Hankel operator $\mathrm{P}_{-} \mathrm{M}_{b} \mathrm{P}_{+}$. Using the calculation in (3.1), we can see that

$$
\left\|\mathrm{P}_{-} \mathrm{M}_{b} \mathrm{P}_{+}\right\|_{p \rightarrow p}=\sup _{\varphi \in H^{p}} \sup _{\psi \in H^{p^{\prime}}} \int\left(\mathrm{P}_{-} b\right) \cdot \varphi \psi d x
$$

But, $H^{1}=H^{p} \cdot H^{p^{\prime}}$, so this last quantity is $\left\|\mathrm{P}_{-} b\right\|_{\mathrm{BMO}}$.

One direction in which this result extends is for the commutator to characterize a broad array of function spaces. The genesis of this theme is the very interesting article of Coifman, Rochberg and Weiss [12], which consider the instance of commutators of $\mathrm{M}_{b}$ and Reisz transforms.

Subsequently, it turns out that one has the equivalence

$$
\left\|\left[\mathrm{M}_{b}, \mathrm{H}\right]\right\|_{X \rightarrow Y} \simeq\|b\|_{Z}
$$

for a range of spaces $X, Y$, and function spaces $Z$. Various Lipschitz classes can be characterized this way; further generalizations can be stated in terms of various Besov and Treibel Lizorkin spaces. Moreover, the Hilbert transform can be replaced by other operators, such as the fractional integral operators. There is a significant literature here, of which we cite Chanillo [11], Cruz-Uribe and Fiorenza [21], as well as the references in the article of the author [40] which is a first step in extending some of these results to higher parameter settings.

Another direction is to abandon the chance of characterizing function spaces, replacing the Hilbert transform by, say, a Calderón Zygmund operator T. The method of choice in such generalizations is the use of the sharp function:

$$
\left(\left[\mathrm{M}_{b}, \mathrm{~T}\right] f\right)^{\sharp} \lesssim \mathrm{M} f,
$$

where on the right we intend $M$ to be an appropriate maximal function. This method was (to the best of my knowledge) first used on this problem by Coifman, Rochberg and Weiss [12], and since then has been used by a wide variety of authors.

This method has difficulties in being generalized to the product setting; we ask the reader's patience for not defining precisely what the sharp function is. ${ }^{2}$

\footnotetext{
${ }^{2}$ A paper of R. Fefferman [23] indicates a certain extension sharp function to the two parameter product setting. The difficulty of a similar extension to a three parameter setting centers around the tenuous relationship between rectangular BMO and BMO in three and higher parameters. See however [66].
} 
There is an alternate method, which is of interest as it highlights the role of paraproducts in these questions, and permits a generalization to the higher parameter setting. See the author's paper [40].

3.2. Paraproducts and Commutators. We describe how to write the commutator $\left[\mathrm{M}_{b}, \mathrm{H}\right]$ as a sum of two paraproducts. From this, appropriate upper bounds on the norms of the commutator can be given. In order to keep the exposition as simple as possible, we will rely on particular properties of the Hilbert transform. Yet, the method is flexible, and can apply to a wide variety of operators.

We return to the Haar basis on the real line. For convenience, set

$$
g_{I}=-h_{I_{\text {left }}}+h_{I_{\text {right }}}, \quad I \in \mathcal{D} .
$$

Here, we are using obvious notation to refer to the left and right halves of the dyadic interval $I$, which again are dyadic. And define an operator by $\mathrm{G} f=\sum_{I \in \mathcal{D}}\left\langle f, h_{I}\right\rangle g_{I}$.

Now, it is well known that the Hilbert transform is nearly diagonalized in a wavelet basis. Yet, since the Hilbert transform has odd kernel, it is is not appropriate to decompose with a 'even' choice of basis. Clearly, $g_{I}$ is an 'odd' version of the Haar function $h_{I}$, so $\mathrm{G}$ is a bit like the Hilbert transform. Of course it lacks translation and dilation invariance. It is a very nice observation of S. Petermichl [58] (also see [59]) that these are the only properties missing. Namely, we have

3.6. Proposition (Petermichl [58]). The operator below is a non zero multiple of the Hilbert transform.

$$
\lim _{Y \rightarrow \infty} \int_{0}^{Y} \int_{1}^{2} \operatorname{Tr}_{-y} \operatorname{Dil}_{1 / s}^{(2)} \operatorname{GDil}_{s}^{(2)} \operatorname{Tr}_{y} \frac{d y}{y} \frac{d s}{s}
$$

Proof. Observe that the limit

$$
\lim _{Y \rightarrow \infty} \int_{0}^{Y} \int_{1}^{2} \operatorname{Tr}_{-y} \operatorname{Dil}_{1 / s}^{(2)} \mathrm{G}_{\varphi} \operatorname{Dil}_{s}^{(2)} \operatorname{Tr}_{y} \frac{d y}{y} \frac{d s}{s}=\Lambda \varphi
$$

exists for Schwartz functions $\varphi$. Moreover, as $G$ is a bounded operator on $L^{2}$, we conclude that $\Lambda$ is also bounded on $L^{2}$.

Due to the limiting procedure, one sees that $\Lambda$ is a translation invariant operator. The average over dilations is taken with respect to Haar measure for the dilation group, hence $\Lambda$ is also invariant with respect to dilations. It is a classical fact that a bounded linear operator, invariant with respect to translations and dilations is a linear combination of the Identity operator and the Hilbert transform.

Clearly, $\Lambda \mathbf{1}=0$. That is, $\Lambda$ is a multiple of the Hilbert transform. And so we should check that it is a not the zero operator. But one can check directly that $\Lambda$ applied to the Dirac 
measure at the origin is

$$
\Lambda \delta_{0}(x) \simeq x^{-1}
$$

Hence it is a multiple of the Hilbert transform.

3.8. Proposition. The commutator $\left[\mathrm{M}_{b}, \mathrm{G}\right]$ can be written as a linear combination of paraproducts, or paraproducts composed with $\mathrm{G}$. In particular, the commutator is bounded on $L^{2}$, when $b \in \mathrm{BMO}$.

Proof. We use the notation $\psi \otimes \varphi$ to denote the rank one linear operator

$$
\psi \otimes \varphi(f)=\psi\langle\varphi, f\rangle \text {. }
$$

We will expand the symbol $b$ in the Haar basis. $\mathrm{G}$ is an explicit sum over rank one operators as above, and we will make an computation of commutators for Haar functions. As such, it is convenient to split the operator $G$ into $G_{\text {left }}$ and $G_{\text {right }}$, where we define

$$
\mathrm{G}_{\text {left }} \stackrel{\text { def }}{=} \sum_{I} h_{I_{\text {left }}} \otimes h_{J}
$$

with a similar definition for $\mathrm{G}_{\text {right }}$. Below we will only consider the 'left' version.

We are lead to expand the commutators

$$
\begin{aligned}
{\left[\mathrm{M}_{h_{I}}, h_{J_{\text {left }}} \otimes h_{J}\right] } & =\left(h_{I} h_{J_{\text {left }}}\right) \otimes h_{J}-h_{J_{\text {left }}} \otimes\left(h_{I} h_{J}\right) \\
& =|J|^{-1 / 2} \begin{cases}0 & I \cap J=\emptyset, \quad J \subsetneq I \\
\sqrt{2}\left[h_{J_{\text {left }}}^{1} \otimes h_{J} \pm h_{J_{\text {left }}} \otimes h_{J_{\text {left }}}\right] & I=J_{\text {left }} \\
h_{J_{\text {left }}} \otimes h_{J_{\text {right }}} & I=J_{\text {right }} \\
-\sqrt{2} h_{J_{\text {left }}} \otimes h_{J}-h_{J_{\text {left }}} \otimes h_{J}^{1} & I=J \\
\sqrt{2} h_{I} \otimes h_{J}-h_{J_{\text {left }}} \otimes h_{I} & I \subsetneq J_{\text {left }} .\end{cases}
\end{aligned}
$$

In this computation, we note that there are two conditions that lead to the commutator being zero. The first is a trivial localization condition, $I \cap J=\emptyset$. The second condition, $J \subsetneq I$, is an essential cancellation condition coming from commutator.

All other terms lead to a paraproduct term, although some of these paraproducts are trivial, in that all relevant functions have a zero. Apply this computation to the commutator in question, expanding as

$$
\left[\mathrm{M}_{b}, \mathrm{G}_{\text {left }}\right]=\sum_{I, J \in \mathcal{D}}\left\langle b, h_{I}\right\rangle\left[\mathrm{M}_{h_{I}}, h_{J_{\text {left }}} \otimes h_{J}\right]
$$

For instance, from the case of $I=J$, we get

$$
c \sum_{J} \frac{\left\langle b, h_{J}\right\rangle}{\sqrt{|J|}} h_{J_{\text {left }}} \otimes h_{J_{\text {left }}}^{1}
$$


which is a paraproduct operator with symbol $b$. Notice that the ' 1 ' falls on the right side of the tensor product of Haar functions. In considering the case $I=J_{\text {left }}$, we get a paraproduct that is dual to the one above, namely

$$
\sum_{J} \frac{\left\langle b, h_{J_{\text {left }}}\right\rangle}{\sqrt{|J|}} h_{J_{\text {left }}}^{1} \otimes h_{J} .
$$

The other term that arises from the case $I=J_{\text {left }}$ is less singular:

$$
\sum_{J} \frac{\left\langle b, h_{J_{\text {left }}}\right\rangle}{\sqrt{|J|}} h_{J_{\text {left }}} \otimes h_{J}
$$

Here, all functions are Haar functions, that is they have zeros. This case is easier to control.

Let us consider the case of $I \subsetneq J_{\text {left }}$. Observe that we have

$$
\mathrm{G}_{\mathrm{left}}^{*} h_{I}^{1}=-\sum_{I \subsetneq J_{\mathrm{left}}} \sqrt{\frac{|I|}{|J|}} h_{J}
$$

Keeping this in mind, we see that

$$
\sum_{I \subsetneq J_{\text {left }}} \frac{\left\langle b, h_{I}\right\rangle}{\sqrt{|J|}} h_{I} \otimes h_{J}=-\sum_{I} \frac{\left\langle b, h_{I}\right\rangle}{\sqrt{|I|}} h_{I} \otimes\left(G^{*} h_{I}^{1}\right)
$$

That is, we have a composition of $\mathrm{G}^{*}$ and a paraproduct. For the other term associated with this case we have

$$
\sum_{I \subsetneq J_{\text {left }}}\left\langle b, h_{I}\right\rangle \frac{h_{J_{\text {left }}}}{\sqrt{|J|}} \otimes h_{I}
$$

This is dual to the previous case. Our proof is complete.

3.10. Remark. A simpler exposition of this approach can be had for commutators of multiplication operators and fractional integral operators. See Lacey [40]. This provides an alternate proof of a result of Chanillo. This result proves another characterization of BMO in terms of a commutator.

\section{Aspects of Product Hardy Theory}

We describe the elements of product Hardy space theory, as developed by S.-Y. Chang and R. Fefferman [6,7,23-25] as well as Journé [35,36]. By this, we mean the Hardy spaces associated with domains like $\mathbb{D} \otimes \mathbb{D}$, with boundary $\mathbb{T} \otimes \mathbb{T}$. In particular, the boundary is flat, and while we work with several variables, we are very far from the pseudoconvex case. 
We view $\mathbb{R}^{d}$ as a tensor product of one dimensional spaces. In particular, previously, we used the splitting of $L^{2}(\mathbb{R})=H^{2}\left(\mathbb{C}_{+}\right) \oplus H_{-}^{2}\left(\mathbb{C}_{+}\right)$. This leads to a decomposition of

$$
L^{2}\left(\mathbb{R}^{d}\right)=\bigotimes_{j=1}^{d} L^{2}(\mathbb{R})=\bigotimes_{j=1}^{d} H^{2}\left(\mathbb{C}_{+}\right) \oplus H_{-}^{2}\left(\mathbb{C}_{+}\right),
$$

into $2^{d}$ components.

To describe them, let us set $\mathrm{P}_{ \pm, j}$ to be the one dimensional Fourier projection operator $\mathrm{P}_{ \pm}$ acting on the $j$ th coordinate. For $\sigma \in\{-,+\}^{d}$, set

$$
\mathrm{P}_{\sigma}=\bigotimes_{j=1}^{d} \mathrm{P}_{\sigma(j), j}
$$

Likewise, we set $H_{\sigma}^{2}\left(\mathbb{C}_{+}^{d}\right)$ to be the range of the orthogonal projection $\mathrm{P}_{\sigma}$. We then have

$$
L^{2}\left(\mathbb{R}^{d}\right)=\bigoplus_{\sigma \in\{+,-\}^{d}} H_{\sigma}^{2}\left(\mathbb{C}_{+}^{d}\right)
$$

Among these $2^{d}$ Hardy spaces, we distinguish $H_{\oplus}^{2}\left(\mathbb{C}_{+}^{d}\right)$ in which $\sigma \equiv+$, and likewise for $H_{\ominus}^{2}\left(\mathbb{C}_{+}^{d}\right)$. The corresponding orthogonal projections are $\mathrm{P}_{\oplus}$ and $\mathrm{P}_{\ominus}$.

Functions $f$ in this space are defined on $\mathbb{R}^{d}$. $\mathbb{R}^{d}$ is viewed as the boundary of the "upper half space'

$$
\mathbb{C}_{+}^{d}=\prod_{j=1}^{d}\{z \in \mathbb{C}: \operatorname{Re}(z)>0\}
$$

And we require that there is a function $F: \mathbb{C}_{+}^{d} \longrightarrow \mathbb{C}$ that is holomorphic in each variable separately, and

$$
f(x)=\lim _{\|y\| \rightarrow 0} f\left(x_{1}+i y_{1}, \ldots, x_{d}+i y_{d}\right) .
$$

The norm of $f$ is taken to be

$$
\|f\|_{H_{\oplus}^{p}\left(\mathbb{C}_{+}^{d}\right)}=\lim _{y_{1} \downarrow 0} \cdots \lim _{y_{d} \downarrow 0}\left\|F\left(x_{1}+y_{1}, \ldots, x_{d}+y_{d}\right)\right\|_{L^{1}\left(\mathbb{R}^{d}\right)}
$$

4.1. Remark. The (real) Hardy space $H^{1}\left(\mathbb{R}^{d}\right)$ typically denotes the class of functions with the norm

$$
\|f\|_{1}+\sum_{j=1}^{d}\left\|\mathrm{R}_{j} f\right\|_{1}
$$

where $\mathrm{R}_{j}$ denote the Reisz transforms. This space is invariant under the one parameter family of isotropic dilations, while $H^{1}\left(\mathbb{C}_{+}^{d}\right)$ is invariant under dilations of each coordinate separately. That is, it is invariant under a $d$ parameter family of dilations. That is why we refer to 'multiparameter' theory, or ' $d$ parameters.' 
As before, the real $H^{1}$, Re $H^{1}\left(\mathbb{C}_{+}^{d}\right)$ has a variety of equivalent norms, in terms of square functions, maximal functions and Hilbert transforms. For our discussion of paraproducts, it is appropriate to make some definitions of translation and dilation operators which extend the definitions in (2.2) - (2.4). (Indeed, here we are adopting broader notation than we really need, in anticipation of a discussion of multiparameter paraproducts.) Define

$$
\begin{aligned}
\operatorname{Tr}_{y} f(x-y) & \stackrel{\text { def }}{=} f(x-y), \quad y \in \mathbb{R}^{d}, \\
\operatorname{Dil}_{t_{1}, \ldots, t_{d}}^{p} f\left(x_{1}, \ldots, x_{d}\right) & \stackrel{\text { def }}{=}\left(t_{1} \cdots t_{d}\right)^{-1 / p} f\left(x_{1} / t_{1}, \ldots, x_{d} / t_{d}\right), \quad t_{1}, \ldots, t_{d}>0 \\
\operatorname{Dil}_{R}^{p} & \stackrel{\text { def }}{=} \operatorname{Tr}_{c(R)} \operatorname{Dil}_{\left|R_{1}\right|, \ldots,\left|R_{d}\right|}^{p} .
\end{aligned}
$$

In the last definition $R=R_{1} \times \cdots \times R_{d}$ is a rectangle, and the dilation incorporates the locations and scales associated with $R . c(R)$ is the center of $R$.

Let $\mathcal{D}^{d}=\mathcal{D} \times \cdots \times \mathcal{D}$ denote the $d$ fold product of the dyadic intervals. These are the dyadic rectangles in $\mathbb{R}^{d}$. For a non negative bump function $\varphi^{1}$ with $\int \varphi^{1} d x=1$, define the (strong) maximal function by

$$
\mathrm{M} \cdots \mathrm{M} f(x)=\sup _{R \in \mathcal{D}^{d}} \operatorname{Dil}_{R}^{2} \varphi^{1}(x)\left\langle f, \operatorname{Dil}_{R}^{2} \varphi^{1}\right\rangle
$$

We use the superscript on $\varphi^{1}$ to indicate that it has a non zero integral.

Fix a bump function $\varphi^{0}$ so that

$$
\varphi^{0}\left(x_{1}, \ldots, x_{d}\right)=\prod_{j=1}^{d} \varphi\left(x_{j}\right)
$$

where $\int_{\mathbb{R}} \varphi d x=0$. Then set an analog of the Littlewood Paley square function to be

$$
\mathrm{S} \cdots \mathrm{S} f(x)=\left[\sum_{R \in \mathcal{D}^{d}}\left[\operatorname{Dil}_{R}^{2} \varphi^{0}(x)\right]^{2}\left|\left\langle f, \operatorname{Dil}_{R}^{2} \varphi^{0}\right\rangle\right|^{2}\right]^{1 / 2}
$$

4.5. Theorem. All of the norms below are equivalent, and can be used as a definition of real $\operatorname{Re} H^{1}\left(\mathbb{C}_{+}^{d}\right)$.

$$
\begin{gathered}
\|\mathrm{M} \cdots \mathrm{M} f\|_{1}, \quad\|\mathrm{~S} \cdots \mathrm{S} f\|_{1}, \quad \sum_{\sigma \in\{0,1\}^{d}}\left\|\mathrm{P}_{\sigma} f\right\|_{1}, \\
\sum_{j=1}^{d} \sum_{\mathrm{A}_{j} \in\left\{\mathrm{I}_{\mathrm{H}}\right\}}\left\|\prod_{j=1}^{d} \mathrm{~A}_{j} f\right\|_{1} .
\end{gathered}
$$

In the last line, we are summing over all choices of operators $\mathrm{A}_{j}$ being either the identity operator, or $\mathrm{H}_{j}$, the Hilbert transform computed in the $j$ th direction. 
4.1. $\mathbf{B M O}\left(\mathbb{C}_{+}^{d}\right)$. The dual of the real Hardy space is $\operatorname{Re}^{1}\left(\mathbb{C}_{+}^{d}\right)^{*}=\operatorname{BMO}\left(\mathbb{C}_{+}^{d}\right)$, the $d$-fold product BMO space. It is a Theorem of S.-Y. Chang and R. Fefferman [7] that this space has a characterization in terms of the product Carleson measure introduced above. We need the product wavelet basis. For a rectangle $R=\prod_{j=1}^{d} R_{(j)} \in \mathcal{D}^{d}$ set

$$
w_{R}\left(x_{1}, \ldots, x_{d}\right)=\prod_{j=1}^{d} w_{R_{(j)}}\left(x_{j}\right)=\operatorname{Dil}_{R}^{2} w_{[0,1]^{d}}(x)
$$

The basis $\left\{w_{R}: R \in \mathcal{D}^{d}\right\}$ is the $d$-fold tensor product of the wavelet basis. We use the same notation $w_{R}$ and $v_{R}$ for the Meyer wavelet basis, and the analytic Meyer wavelet basis. Define

$$
\|b\|_{\mathrm{BMO}\left(\mathbb{R}^{d}\right)} \simeq \sup _{U \subset \mathbb{R}^{d}}\left[|U|^{-1} \sum_{R \subset U}\left|\left\langle b, w_{R}\right\rangle\right|^{2}\right]^{1 / 2}
$$

where we have replaced the Haar wavelets by the Meyer wavelets on the right.

It is the Theorem of Chang and Fefferman that

4.7. Theorem. We have the equivalence of norms

$$
\|f\|_{\left(\operatorname{Re} H^{1}\left(\mathbb{C}_{+}^{d}\right)\right)^{*}} \simeq\|f\|_{\mathrm{BMO}\left(\mathbb{R}^{d}\right)}
$$

That is, $\mathrm{BMO}\left(\mathbb{R}^{d}\right)$ is the dual to $\operatorname{Re} H^{1}\left(\mathbb{C}_{+}^{d}\right)$.

To define analytic $\mathrm{BMO}\left(\mathbb{C}_{+}^{d}\right)$, it suffices to replace the Meyer wavelets above by the analytic Meyer wavelets.

4.2. Journé's Lemma. The explicit definition of BMO in (4.6) is quite difficult to work with. In the first place, it is not an intrinsic definition, in that one needs some notion of wavelet to define it. Secondly, the supremum is over a very broad class of objects: All subsets of $\mathbb{R}^{d}$ of finite measure. There are simpler definitions, (that unfortunately are not intrinsic) that in particular circumstances are sufficient.

For our purposes, there are two appropriate definitions. Set $\|f\|_{\mathrm{BMO}(\mathrm{rec})}$ to be the supremum in (4.6), but with the important restriction that the sets $U$ are taken to be rectangles. Historically, this was the first natural guess for the correct definition of $\operatorname{BMO}\left(\mathbb{C}_{+}^{d}\right)$. But, in a key moment, L. Carleson [5] produced examples of functions which acted as linear functionals on $\mathrm{H}^{1}\left(\mathbb{C}_{+}^{d}\right)$ with norm one, yet had arbitrarily small BMO(rec) norm. This example is recounted at the beginning of $\mathrm{R}$. Fefferman's article [25].

Despite this fact, Journé Lemma shows that in certain circumstances, the rectangular $\mathrm{BMO}(\mathrm{rec})$ norm can dominate the $\mathrm{BMO}\left(\mathbb{C}_{+}^{d}\right)$ norm. Let us state this Lemma in the case of $\mathbb{C}_{+}^{2}$ before moving to the more sophisticated variants that we will need in three and higher parameters. 
Given a set $U \subset \mathbb{R}^{2}$ of finite measure, let

$$
\begin{gathered}
\operatorname{Emb}(R ; U)=\sup \{\mu>1: \mu R \subset V\} \\
V \stackrel{\text { def }}{=}\left\{\mathrm{M} \mathrm{M} \mathbf{1}_{\left\{\mathrm{M} \mathrm{M} \mathbf{1}_{U}>\frac{1}{2}\right\}}>\frac{1}{2}\right\}
\end{gathered}
$$

This is defined for rectangles $R \subset U$, where $\mu R$ denotes the rectangle with the same center as $R$, which is dilated by a factor of $\mu$ in all directions. Notice that we have $|V| \lesssim|U|$, and that $V$ is a natural 'dilate of $U$.' The function $\operatorname{Emb}(R ; U)$ is a measure of how deeply embedded $R$ is inside of $U$.

A key distinction in two and higher parameters concerns collection of rectangles $\{R: R \subset$ $U, \operatorname{Emb}(R ; U) \simeq \mu\}$. This collection of rectangles is not pairwise disjoint, but their overlap is, in appropriate sense, at worst logarithmic in $\mu$. A formulation of this principle is easiest in two parameters.

4.8. Lemma (Journé's Lemma [35] in $\mathbb{R}^{2}$ ). For any function $f$, and $\epsilon>0$, we have the inequality below valid for all sets $U \subset \mathbb{R}^{2}$ of finite measure.

$$
\left\|\sum_{R \subset U} \operatorname{Emb}(R ; U)^{-\epsilon}\left\langle f, w_{R}\right\rangle w_{R}\right\|_{\mathrm{BMO}\left(\mathbb{R}^{d}\right)} \lesssim\|f\|_{\mathrm{BMO}(\mathrm{rec})}
$$

The implied constant depends only on $\epsilon>0$.

Notice that the last inequality is that the BMO norm is dominated by the (generally smaller) BMO(rec) norm. Carleson's examples show that this inequality is false if we do not 'dampen' the wavelet coefficients in some way. Journé's insight is that this can be done with the geometric notion of the enlargement term.

We will need this observation in the case of $\mathbb{C}_{+}^{2}$. But, the rectangular norm is ill suited to our needs in three and higher dimensions. We make this definition, which reduces to rectangular BMO in dimension 2.

Say that a collection of rectangles $\mathcal{U} \subset \mathcal{D}^{d}$ has $d-1$ parameters iff there is a choice of coordinate $j$ so that for all $R, R^{\prime} \in \mathcal{U}$ we have $R_{(j)}=R_{(j)}^{\prime}$, that is the $j$ th coordinate of the rectangles agree.

We then define

$$
\|f\|_{\mathrm{BMO}_{-1}\left(\mathbb{R}^{d}\right)} \stackrel{\text { def }}{=} \sup _{\substack{\mathcal{U} \text { has } d-1 \\ \text { parameters }}}\left[|\operatorname{sh}(\mathcal{U})|^{-1} \sum_{R \in \mathcal{U}}\left|\left\langle f, w_{R}\right\rangle\right|^{2}\right]^{1 / 2}
$$

A collection of rectangles has a shadow given by $\operatorname{sh}(\mathcal{U}) \stackrel{\text { def }}{=} \bigcup\{R: R \in \mathcal{U}\}$. Observe that in $d=2$ this reduces to the rectangular BMO definition. We use the -1 subscript to indicate that we have 'lost one parameter' in the definition. 
The extension of Journé's Lemma that we need replaces the BMO(rec) norm by this $\mathrm{BMO}_{-1}\left(\mathbb{C}_{+}^{d}\right)$ norm. Yet one more refinement is essential for our needs, that the 'dilate' of the set $U$ be taken with considerably more care, and in particular should be just a little bit bigger than $U$ in measure, see (4.12).

4.10. Lemma (Journé's Lemma in $d-1$ parameters). For all $\eta>0$, and collections of rectangles $\mathcal{U}$ whose shadow has finite measure, we can construct $V \supset \operatorname{sh}(\mathcal{U})$ and a function $\mathrm{Emb}: \mathcal{U} \longrightarrow[1, \infty)$ so that

$$
\begin{gathered}
\operatorname{Emb}(R) \cdot R \subset V, \quad R \in \mathcal{U} \\
|V|<(1+\eta)|\operatorname{sh}(U)| \\
\left\|\sum_{R \subset U} \operatorname{Emb}(R ; U)^{-2 d}\left\langle f, w_{R}\right\rangle w_{R}\right\|_{\mathrm{BMO}\left(\mathbb{R}^{d}\right)} \leq K_{\eta}\|f\|_{\mathrm{BMO}_{-1}\left(\mathbb{R}^{d}\right)}
\end{gathered}
$$

The last inequality holds for all functions $f$, with the constant $K_{\eta}$ depending only on $\eta$.

Notice that the power on the embeddedness term in (4.13) is quite large, twice the number of parameters. Also, concerning the conclusions, if we were to take $\operatorname{Emb}(R) \equiv 1$, then certainly the first conclusion (4.11) would be true. But, the last conclusion would be false for the Carleson examples in particular. This choice is obviously not permitted in general.

The formulations of Journé's Lemma given here are not the typical ones found in Journé's original Lemma, or J. Pipher's extension to three dimensional case. These papers give the more geometric formulation of these Lemmas, and J. Pipher's article implicitly contains the geometric formulation needed to prove the Lemma above (provided one is satisfied with the estimate $|V| \lesssim|\operatorname{sh}(\mathcal{U})|)$. See Pipher [60]. Lemma 4.10, as formulated above, was found in Lacey and Terwelleger [38]; the two dimensional variant (which is much easier) appeared in Lacey and Ferguson [27]. The paper of Cabrelli, Lacey, Molter and Pipher [4] is a comprehensive survey of issues related to Journé's Lemma. See in particular Sections 2 and 4. We refer the reader to it for more information on this subject.

\section{Multiparameter Paraproducts}

We now consider paraproducts formed over sums of dyadic rectangles in $\mathbb{R}^{d}$. Let us say that a function $\varphi$ is adapted to a rectangle $R=\otimes_{j=1}^{d} R_{j}$ iff $\varphi\left(x_{1}, \ldots, x_{d}\right)=\prod_{j=1}^{d} \varphi_{j}\left(x_{j}\right)$, with each $\varphi_{j}$ adapted to the interval $R_{j}$ in the sense of (2.17).

Our paraproducts are of the same general form

$$
\mathrm{B}\left(f_{1}, f_{2}\right) \stackrel{\text { def }}{=} \sum_{R \in \mathcal{R}} \frac{\varphi_{3, R}}{|R|^{\frac{1}{2}}} \prod_{v=1}^{2}\left\langle f_{v}, \varphi_{v, R}\right\rangle .
$$


Here, we let $\mathcal{R} \stackrel{\text { def }}{=} \mathcal{D}^{d}$ be the class of dyadic rectangles.

The Theorem in this setting is

5.1. Theorem. Let $1<p<\infty$, and $J \subset\{1, \ldots, d\}$. Assume that for each choice of coordinate $1 \leq j \leq d$, and $v=1$

$$
\int_{\mathbb{R}} \varphi_{v, R}\left(x_{1}, x_{2}, \ldots, x_{n}\right) d x_{j}=0, \quad \text { for all } x_{k} \text { with } k \neq j \text { and all } R .
$$

In addition, for each $1 \leq j \leq d$ and all $R \in \mathcal{D}^{d}$, assume that the condition above holds for $\varphi_{v, R}$, where $v=2$ if $j \in J$ and $v=3$ if $j \notin J$. Then, we have the inequality

$$
\mathrm{B}: \operatorname{BMO}\left(\mathbb{C}_{+}^{d}\right) \times L^{p} \longrightarrow L^{p} .
$$

We are not stating this result in greatest generality. It was first discussed in the the paper of Journé [36]. Recently, the result has received new attention, and extension. See Muscalu, Pipher, Tao and Thiele [44,45]. Our discussion is drawn from Lacey and Metcalfe [37]. And in particular, this last paper proves this Theorem.

The critical distinction comes from the assumption about the zeros, (5.2). We need zeros in every coordinate on the functions that land on the $\mathrm{BMO}\left(\mathbb{C}_{+}^{d}\right)$ function. There is one more zero in each coordinate, and they can be split up between the second and third functions.

Notice that there are many different types of paraproducts. The first case, with the greatest similarity to the one parameter case, is where we have, for example, $x_{j}$ zeros in first and second positions for all $1 \leq j \leq d$. The other cases do not have a proper analog in the one parameter case.

We will have need of paraproducts which are presented in a somewhat different way, in analogy to Theorem 2.23. We make some definitions. For $\vec{s} \in \mathbb{Z}^{d}$, let us set

$$
\Delta \mathrm{U}_{\vec{j}}=\sum_{\substack{R \in \mathcal{D}^{d} \\\left|R_{s}\right|=2^{j_{s}}, 1 \leq s \leq d}} u_{R} \otimes u_{R}
$$

For a subset of coordinates $J \subset\{1, \ldots, d\}$ set

$$
\mathrm{U}_{\vec{\jmath}, J} \stackrel{\text { def }}{=} \sum_{\substack{\vec{k} \in \mathbb{Z}^{d} \\ k_{s}=j_{s}, s \in J \\ k_{s} \geq j_{s}, s \notin J}} \Delta \mathrm{U}_{\vec{k}}
$$

For those coordinates $s \in J$, we take the wavelet projection onto that scale, while for those coordinates $s \notin J$, we sum over all larger scales.

Write $R^{\prime} \lesssim J$ iff $\left|R_{s}^{\prime}\right| \leq\left|R_{s}\right|$ for $s \notin J$ and $\left|R_{s}^{\prime}\right|=\left|R_{s}\right|$ for $s \in J$. 
5.4. Theorem. For all $J \subset\{1, \ldots, d\}$, and $\vec{k} \in \mathbb{Z}^{d}$ with $\|\vec{k}\|_{\infty} \leq 8$, we have

$$
\left\|\sum_{\vec{\jmath} \in \mathbb{Z}^{d}}\left(\Delta \mathrm{U}_{\vec{\jmath}, J} b\right) \cdot \overline{\mathrm{U}_{\vec{\jmath}+\vec{k}, J} \varphi}\right\|_{2} \lesssim\|b\|_{\mathrm{BMO}\left(\mathbb{C}_{+}^{d}\right)}\|\varphi\|_{2}
$$

Moreover, suppose we have the following separation condition: Fix an integer $A>0$. Suppose that

$$
\text { if }\left\langle b, u_{R^{\prime}}\right\rangle \neq 0,\left\langle\varphi, u_{R}\right\rangle \neq 0 \text { with } R^{\prime} \lesssim J \text {, then } A R \cap R^{\prime}=\emptyset .
$$

We then have the estimate

$$
\left\|\sum_{\vec{\jmath} \in \mathbb{Z}^{d}}\left(\Delta \mathrm{U}_{\vec{\jmath}, J} b\right) \cdot \overline{\mathrm{U}_{\vec{\jmath}+\vec{k}, J} \varphi}\right\|_{2} \lesssim A^{-100 d}\|b\|_{\mathrm{BMO}\left(\mathbb{C}_{+}^{d}\right)}\|\varphi\|_{2}
$$

Implied constants are independent of the choice of $\vec{k}$.

Proof. The method of proof is quite similar to that of Theorem 2.23.

We treat a special case with a brute force approach. Consider

$$
\sum_{\vec{\jmath} \in \mathbb{Z}^{d}}\left(\Delta \mathrm{U}_{\vec{\jmath}} b\right) \cdot\left(\overline{\Delta \mathrm{U}_{\vec{\jmath}+\vec{k}} \varphi}\right), \quad k \in \mathbb{Z}^{d},\|\vec{k}\|_{\infty} \leq 8 .
$$

We claim that the two estimates of the Theorem hold for these operators.

Fix an integer $B \geq 2$ Let $\pi: \mathcal{D}^{d} \longrightarrow \mathcal{D}^{d}$ be a map so that for all $R \in \mathcal{D}^{d}$ we have $2^{k_{s}}\left|R_{s}\right|=\left|\pi(R)_{s}\right|$ for $1 \leq s \leq d$. In addition, the distance between $R$ and $\pi(R)$ is essentially constant. Namely,

$$
B^{-1} \leq \mathrm{M} \cdots \mathrm{M} \mathbf{1}_{R}\left(c(\pi(R)) \leq(B-1)^{-1}\right.
$$

(In the current setting, it is most natural to use the maximal function to measure distances.) Then, the sum

$$
\sum_{R \in \mathcal{D}^{d}} \frac{\left\langle b, u_{R}\right\rangle}{\sqrt{|R|}} \overline{\left\langle\varphi, u_{\pi(R)}\right\rangle} \cdot \sqrt{|R|} u_{R} \overline{u_{\pi(R)}}
$$

is a paraproduct, with zeros in all coordinates for both $b$ and $\varphi$. This is not necessarily the case of the third place, but $B^{200 d} \sqrt{|R|} u_{R} \overline{u_{\pi(R)}}$ is adapted to $R$ with constant independent of $B$. To prove (5.5), we then sum over $B \geq 1$; to prove (5.6), sum over $B \geq A$.

We recall the 'father wavelet' $W$ from the proof of Theorem 2.23. For a subset of coordinates $J \subset\{1, \ldots, d\}$ we set

$$
W_{R, J}\left(x_{1}, \ldots, x_{d}\right) \stackrel{\text { def }}{=} \prod_{s \in J} u_{R_{s}}\left(x_{s}\right) \cdot \prod_{s \notin J} W_{R_{s}}\left(x_{s}\right)
$$

thus, in the coordinates in $J$ we take an analytic Meyer wavelet, and for those coordinates not in $J$ we take a father wavelet. 
Observe that

$$
\mathrm{U}_{\vec{\jmath}, J}=\left[\sum_{\substack{R \in \mathcal{D}^{d} \\\left|R_{s}\right|=2^{j_{s}}}} W_{R, J} \otimes W_{R, J}\right] \circ \mathrm{P}_{\oplus} .
$$

For $\overrightarrow{9}=(9, \ldots, 9)$, we need to provide the two bounds for the Theorem for

$$
\sum_{\vec{\jmath} \in \mathbb{Z}^{d}}\left(\Delta \mathrm{U}_{\vec{\jmath}} b\right) \cdot \overline{\mathrm{U}_{\vec{\jmath}+\overrightarrow{9}, J} \mathrm{P}_{\oplus}}
$$

For an integer $A$, and map $\pi$ as above, it suffices to consider the sum

$$
\begin{gathered}
\sum_{R \in \mathcal{D}^{d}} \frac{\left\langle b, u_{R}\right\rangle}{\sqrt{|R|}} \overline{\left\langle\varphi, W_{\pi(R), J}\right\rangle} \psi_{R, J}, \\
\psi_{R, J} \stackrel{\text { def }}{=} \sqrt{|R|} u_{R} \cdot \mathrm{W}_{\pi(R), J} .
\end{gathered}
$$

This is a paraproduct. Note that for the function $b$, we have zeros in all coordinates; for the function $\varphi$, we have zeros in coordinates $s \notin J$; the function $\psi_{R, J}$ has zeros in those coordinates $s \in J$. Finally, $B^{200 d} \psi_{R, J}$ is adapted to $R$ with constants that are independent of $B$ or the choice of $\pi$. Thus, the two claimed inequalities of the Theorem hold for these sums, and this completes the proof of the Theorem.

\section{Nehari Theorem in Several Variables} by

The Hankel operators we are are concerned with are maps from $H_{\oplus}^{2}\left(\mathbb{C}_{+}^{d}\right)$ to $H_{\oplus}^{2}\left(\mathbb{C}_{+}^{d}\right)$ given

$$
\mathrm{H}_{b} \varphi \stackrel{\text { def }}{=} \mathrm{P}_{\oplus} \mathrm{M}_{b} \bar{\varphi}
$$

This definition only depends upon $\mathrm{P}_{\oplus} b$.

6.2. Remark. These are the 'little' Hankel operators, in that we are taking the 'smallest' reasonable projection above. To define the 'big' Hankel operators, one would replace $\mathrm{P}_{\ominus}$ above by $\mathrm{I}-\mathrm{P}_{\oplus}$. We refer the reader to Cotlar and Sadosky [17] for the theory of these 'big' Hankel operators.

The Nehari Theorem in this context is:

Multiparameter Nehari Theorem ([27,38]). We have the equivalence

$$
\left\|\mathrm{H}_{b}\right\| \simeq\left\|\mathrm{P}_{\oplus} b\right\|_{\mathrm{BMO}\left(\mathbb{C}_{+}^{d}\right)}
$$

where the latter space is S.-Y. Chang and R. Fefferman BMO, the dual to the Hardy space $H^{1}\left(\mathbb{C}_{+}^{d}\right)$. 
This theorem has equivalent statements; the most obvious of these concerns the multiparameter commutator

$$
\mathrm{C}(b, f) \stackrel{\text { def }}{=}\left[\cdots\left[\mathrm{M}_{b}, \mathrm{H}_{1}\right], \ldots, \mathrm{H}_{d}\right]
$$

where $\mathrm{H}_{j}$ denotes the Hilbert transform computed in the $j$ th coordinate.

Less obviously, there is an an equivalent formulation in terms of factorization. As we have commented, the classical factorization of $H^{1}$ functions given in Proposition 2.11 does not extend to $H^{1}\left(\mathbb{C}_{+}^{d}\right)$. The Nehari theorem is equivalent to weak factorization. The formalization of this is done in terms of a tensor products of $H^{2}\left(\mathbb{C}_{+}^{d}\right)$.

We define a projective tensor product norm by

$$
\|f\|_{H^{2}\left(\mathbb{C}_{+}^{d}\right) \widehat{\otimes} H^{2}\left(\mathbb{C}_{+}^{d}\right)} \stackrel{\text { def }}{=} \inf \left\{\sum_{j}\left\|\varphi_{j}\right\|_{H^{2}\left(\mathbb{C}_{+}^{d}\right)}\|\psi\|_{H^{2}\left(\mathbb{C}_{+}^{d}\right)}: f=\sum_{j} \varphi_{j} \psi_{j}, \varphi_{j}, \psi_{j} \in H^{2}\left(\mathbb{C}_{+}^{d}\right)\right\}
$$

6.4. Theorem. Any one of equivalences of norms below are consequences of the other equivalences.

$$
\begin{aligned}
\left\|\mathrm{H}_{b}\right\| & \simeq\left\|\mathrm{P}_{\oplus} b\right\|_{\mathrm{BMO}\left(\mathbb{R}^{d}\right)} \\
\left\|\mathrm{C}_{b}\right\|_{2 \rightarrow 2} & \simeq\|b\|_{\mathrm{BMO}\left(\mathbb{C}_{+}^{d}\right)} \\
\|f\|_{H^{1}\left(\mathbb{C}_{+}^{d}\right)} & \simeq\|f\|_{H^{2}\left(\mathbb{C}_{+}^{d}\right) \widehat{\otimes} H^{2}\left(\mathbb{C}_{+}^{d}\right)}
\end{aligned}
$$

In the first two, we take the $\mathrm{BMO}\left(\mathbb{R}^{d}\right)$ norm to be real valued $\mathrm{BMO}$. In the second two, $\mathrm{BMO}\left(\mathbb{C}_{+}^{d}\right)$ is analytic BMO.

The last equivalence of norms is the weak factorization statement in $H^{1}\left(\mathbb{C}_{+}^{d}\right)$. It explains in part why the factorization proof of the one parameter Nehari theorem is so easy: The factorization property is stronger than Nehari's Theorem.

6.8. Corollary. We have

$$
\left\|\mathrm{H}_{b}\right\|=\inf \left\{\|\beta\|_{\infty}: \mathrm{P}_{\oplus} b=\mathrm{P}_{\oplus} \beta\right\}
$$

Theorem 6.4 was known and elementary; once weak factorization (6.7) is known, Corollary 6.8 is easy. Thus, the Multiparameter Nehari Theorem is the main point. $\mathrm{x}$ The inequality $\left\|\mathrm{H}_{b}\right\| \lesssim\|b\|_{\mathrm{BMO}\left(\mathbb{C}_{+}^{d}\right)}$, turns out to be quite easy - it is a consequence of the trivial inclusion in the weak factorization statement. The issue is to establish the lower bound on the norm of the Hankel operator.

The central difficulty here lies in the subtle nature of BMO in the higher parameter case. The proof we give is an induction on $d$, using weak factorization in $H^{1}\left(\mathbb{C}_{+}^{d-1}\right)$ in a critical moment. Appealing to weak factorization will give us a lower bound in terms of $\mathrm{BMO}_{-1}$. And so we need to 'bootstrap' from this weaker inequality to the stronger inequality. The boostrapping argument appeals to the Journé Lemma. 
It suffices to assume that $b=\mathrm{P}_{\oplus} b \in \mathrm{BMO}\left(\mathbb{C}_{+}^{d}\right)$ is of norm one, and find an absolute lower bound on $\left\|H_{b}\right\|$. We begin by using the induction hypothesis to establish

$$
\left\|H_{b}\right\| \gtrsim\|b\|_{\mathrm{BMO}_{-1}\left(\mathbb{C}_{+}^{d}\right)}
$$

where the latter norm is BMO norm 'with one less parameter' defined in (4.9). Thus, we are free to impose the additional hypothesis that $\|b\|_{\mathrm{BMO}_{-1}\left(\mathbb{C}_{+}^{d}\right)}$ is less than some fixed, absolute constant. Observe that implicitly, this forces $b$ to be the type of functions which Carleson discovered.

Yet, Journé's Lemma gives modest sufficient conditions for this impoverished norm to dominate the true BMO norm. The lower bound for the norm of $\mathrm{H}_{b}$ can then be explicitly estimated as a main term, plus several error terms. Each of the error terms is a paraproduct, which can be controlled with Journé's Lemma and the fact that the improvised norm is small.

Proof of Theorem 6.4. We discuss the proof of Theorem 6.4 and Corollary 6.8. Observe that the computation (3.1) is quite general. In the language we have introduced above, it shows immediately that

$$
\left\|\mathrm{H}_{b}\right\| \simeq\left\|\mathrm{P}_{\oplus} b\right\|_{H^{2}\left(\mathbb{C}_{+}^{d}\right) \widehat{\otimes} H^{2}\left(\mathbb{C}_{+}^{d}\right)^{*}}
$$

That is, the Hankel norms are equivalent to the dual norm of the tensor product norm.

The equivalence of (6.5) and (6.7) is then immediate.

Concerning the commutator, and (6.6), as in the one parameter case, the commutator is seen to be a sum of $2^{d}$ Hankel operators. Indeed, for $\sigma \in\{-,+\}^{d}$, consider the composition $\mathrm{C}_{b} \mathrm{P}_{\sigma}$ In the definition of the commutator, we are free to replace the $j$ th Hilbert transform $\mathrm{H}_{j}$ by $\mathrm{P}_{-\sigma(j), j}$, since $H_{j}= \pm\left(\mathrm{I}-2 \mathrm{P}_{-\sigma(j), j}\right)$, and the identity commutes with everything. Thus,

$$
\mathrm{C}_{b} \mathrm{P}_{\sigma}= \pm 2^{d} \mathrm{P}_{-\sigma} \mathrm{M}_{b} \mathrm{P}_{\sigma}
$$

From this, the equivalence of (6.5) and (6.6) is immediate.

Proof of Corollary 6.8. We can assume that the symbol of the Hankel operator $H_{b}$ is in analytic BMO. Then, (6.9) and (6.7) show that $b$ defines a bounded linear functional on $H^{1}\left(\mathbb{C}_{+}^{d}\right) \subset L^{1}\left(\mathbb{R}^{d}\right)$. Appeal to the Hahn Banach Theorem to extend this linear functional to all of $L^{1}\left(\mathbb{R}^{d}\right)$, with the same norm. The Corollary follows. 


\section{Proof of Multiparameter Nehari Theorem}

The upper bound on the norm of a Hankel operator is easy. Observe that, trivially,

$$
H^{2}\left(\mathbb{C}_{+}^{d}\right) \widehat{\otimes} H^{2}\left(\mathbb{C}_{+}^{d}\right) \subset H^{1}\left(\mathbb{C}_{+}^{d}\right)
$$

For the dual spaces, we have the reverse inclusion. In particular, the $\operatorname{BMO}\left(\mathbb{C}_{+}^{d}\right)$ norm is larger than the dual tensor product norm. Thus, by (6.3),

$$
\begin{aligned}
\left\|\mathrm{H}_{b}\right\| & \simeq\left\|\mathrm{P}_{\oplus} b\right\|_{H^{2}\left(\mathbb{C}_{+}^{d}\right) \widehat{\otimes} H^{2}\left(\mathbb{C}_{+}^{d}\right)^{*}} \\
& \lesssim\left\|\mathrm{P}_{\oplus} b\right\|_{\mathrm{BMO}\left(\mathbb{C}_{+}^{d}\right)}
\end{aligned}
$$

Thus, the primary difficulty is in establishing the lower bound on the norm of the Hankel operator.

7.1. The Initial Lower Bound. The proof is by induction on dimension $d$, and we take the classical Nehari Theorem as the base case in the induction. Thus, we assume that (6.3) holds in dimension $d-1 \geq 1$, and prove it in dimension $d$.

Take $b$ to be in analytic $\operatorname{BMO}\left(\mathbb{C}_{+}^{d}\right)$, and of norm one. We recall that this means in particular, that we have

$$
\sup _{U \subset \mathbb{C}_{+}^{d}}|U|^{-1} \sum_{\substack{R \in \mathcal{D}^{d} \\ R \subset U}}\left|\left\langle b, v_{R}\right\rangle\right|^{2}=1,
$$

where we recall that the supremum is over all subsets $U$ of finite measure, and that the functions $v_{R}$ are the analytic Meyer wavelets associated to dyadic rectangles in $\mathbb{C}_{+}^{d}$.

Let us argue that

$$
\left\|\mathrm{H}_{b}\right\| \gtrsim\|b\|_{\mathrm{BMO}_{d-1}\left(\mathbb{C}_{+}^{d}\right)}
$$

This last norm is given in (4.9), and in particular, it is a supremum as in (7.1), with an additional restriction on rectangles that contribute to that sum.

Now, this inequality we are to prove, by (6.9), reduces to showing

$$
\left\|\mathrm{P}_{\oplus} b\right\|_{\left(H^{2}\left(\mathbb{C}_{+}^{d}\right) \hat{\otimes} H^{2}\left(\mathbb{C}_{+}^{d}\right)\right)^{*}} \gtrsim\left\|\mathrm{P}_{\oplus} b\right\|_{\mathrm{BMO}_{d-1}\left(\mathbb{C}_{+}^{d}\right)} .
$$

We can assume that $b=\mathrm{P}_{\oplus} b$ is a Schwartz function, and that $\|b\|_{\mathrm{BMO}_{d-1}\left(\mathbb{C}_{+}^{d}\right)}=1$. Thus, after a permutation of coordinate and a possible dilation, we can take a collection of rectangles $\mathcal{U}$ which achieves the supremum in the $\mathrm{BMO}_{d-1}\left(\mathbb{C}_{+}^{d}\right)$ norm.

In particular, we can assume that

- $|\operatorname{sh}(\mathcal{U})|=1$;

- there is an interval $I$ of length one so that for all $R \in \mathcal{U}$ we have $R_{1}=I$; 
- for $\psi=\sum_{R \in \mathcal{U}}\left\langle b, v_{R}\right\rangle v_{R}$ we have $\langle b, \psi\rangle=1$.

Then, it suffices to see that $\|\psi\|_{H^{2}\left(\mathbb{C}_{+}^{d}\right) \widehat{\otimes} H^{2}\left(\mathbb{C}_{+}^{d}\right)} \lesssim 1$.

Write $x=\left(x_{1}, x_{2}, \ldots, x_{d}\right) \in \mathbb{R}^{d}$ as $\left(x_{1}, x^{\prime}\right)$ with $x^{\prime}=\left(x_{2}, \ldots, x_{d}\right) \in \mathbb{R}^{d-1}$. Each rectangle $R \in \mathcal{U}$ has the same first coordinate. So the first coordinate in the in product that defines the Meyer analytic wavelet $v_{R}$ is independent of $R$. Therefore, we can write $\psi(x)=\psi_{1}\left(x_{1}\right) \psi^{\prime}\left(x^{\prime}\right)$ where $\psi_{1}\left(x_{1}\right) \in H^{1}(\mathbb{R})$ is of norm one. It can written as $\psi_{1}=\alpha \cdot \beta$ with $\alpha_{1}$ and $\beta_{1}$ of $H^{2}(\mathbb{R})$ norm one.

$\psi^{\prime}$ satisfies something similar. Observe that

$$
\left\|\psi^{\prime}\right\|_{H^{1}\left(\mathbb{C}_{+}^{d-1}\right)} \leq|U|^{1 / 2}\left\|\psi^{\prime}\right\|_{2} \leq 1 .
$$

Hence, $\psi^{\prime}$ is in $H^{1}\left(\mathbb{C}_{+}^{d-1}\right)$, and is of norm at most one. In fact, it has norm comparable to one, since by construction $\left\|\psi^{\prime}\right\|_{\mathrm{BMO}\left(\mathbb{C}_{+}^{d-1}\right)} \leq 1$ and $\left\langle\psi^{\prime}, \psi^{\prime}\right\rangle=1$. Thus, by the induction hypothesis, we have

$$
\left\|\psi^{\prime}\right\|_{H^{1}\left(\mathbb{C}_{+}^{d-1}\right)} \simeq\left\|\psi^{\prime}\right\|_{H^{2}\left(\mathbb{C}_{+}^{d-1}\right) \widehat{\otimes} H^{2}\left(\mathbb{C}_{+}^{d-1}\right)} \simeq 1
$$

Thus, $\psi^{\prime}$ can be written as a sum of products of $\alpha_{j}^{\prime} \cdot \beta_{j}^{\prime}$ with

$$
\sum_{j}\left\|\alpha_{j}^{\prime}\right\|_{H^{2}\left(\mathbb{C}_{+}^{d-1}\right)}\left\|\beta_{j}^{\prime}\right\|_{H^{2}\left(\mathbb{C}_{+}^{d-1}\right)} \simeq 1
$$

But then, it is clear that we can write

$$
\psi\left(x_{1}, x^{\prime}\right)=\sum_{j} \alpha\left(x_{1}\right) \alpha_{j}^{\prime}\left(x^{\prime}\right) \cdot \beta\left(x_{1}\right) \beta_{j}^{\prime}\left(x^{\prime}\right)
$$

and so $\|\psi\|_{H^{2}\left(\mathbb{C}_{+}^{d}\right) \widehat{\otimes} H^{2}\left(\mathbb{C}_{+}^{d}\right)} \lesssim 1$.

7.2. The $\mathrm{BMO}\left(\mathbb{C}_{+}^{d}\right)$ lower bound. Our task is to 'bootstrap' from the weaker inequality (7.2). Namely, for an absolute constant $\eta_{-1}$ whose value is to be specified, it suffices to consider Hankel symbols $b$ which satisfy $b=\mathrm{P}_{\oplus} b ; b$ is Schwartz function; $\|b\|_{\mathrm{BMO}\left(\mathbb{C}_{+}^{d}\right)}=1$; and $\|b\|_{\mathrm{BMO}_{-1}\left(\mathbb{C}_{+}^{d}\right)}<\eta_{-1}$. (The subscript ${ }_{-1}$ mimics our notation for the reduced parameter BMO space.)

We show by direct computation that $\left\|\mathrm{H}_{b}\right\| \gtrsim 1$, namely we will apply the Hankel operator to a particular $H^{2}\left(\mathbb{C}_{+}^{d}\right)$ function, and provide a lower bound on the norm of the image.

Here is how we select the test function to apply the Hankel to. Select a collection of rectangles $\mathcal{U}$ which achieve the supremum in the definition of $\mathrm{BMO}\left(\mathbb{C}_{+}^{d}\right)$ norm. Thus,

$$
\sum_{R \in \mathcal{U}}\left|\left\langle b, v_{R}\right\rangle\right|^{2}=|\operatorname{sh}(\mathcal{U})|
$$


Moreover, we can, after taking an appropriate dilation, that $|\operatorname{sh}(\mathcal{U})|=1$, and that if $R \subset$ $\operatorname{sh}(\mathcal{U})$, then $R \in \mathcal{U}$.

The function we apply the Hankel to the wavelet projection of $b$ onto the wavelets associated with $\mathcal{U}, \alpha=\sum_{R \in \mathcal{U}}\left\langle b, v_{R}\right\rangle v_{R}$. Observe that

$$
\begin{aligned}
\left\|\mathrm{H}_{b} \alpha\right\| & =\left\|\mathrm{P}_{\oplus}|\alpha|^{2}\right\|_{2} \\
& \gtrsim\left\||\alpha|^{2}\right\|_{2} \\
& =\|\alpha\|_{4}^{2} \\
& \simeq\left\|\left[\sum_{R \in \mathcal{U}} \frac{\left|\left\langle b, v_{R}\right\rangle\right|^{2}}{|R|} \mathbf{1}_{R}\right]^{1 / 2}\right\|_{4}^{2} \\
& \geq\left[\sum_{R \in \mathcal{U}}\left|\left\langle b, v_{R}\right\rangle\right|^{2}\right]^{1 / 2} \simeq 1 .
\end{aligned}
$$

Here, we are relying on the symmetry of the Fourier transform of positive functions; Littlewood Paley inequalities, to pass to the wavelet square function; that $\mathcal{U}$ has shadow equal to one in measure, and that $L^{4}$ norms dominate $L^{2}$ norms on a probability space. Thus, we have $\left\|\mathrm{H}_{\alpha} \alpha\right\| \geq \eta_{0}>0$, for absolute $\eta_{0}$.

This is in fact our main estimate. Our task is to show that for $\eta_{-1}$ sufficiently small, that we have

$$
\left\|\mathrm{H}_{b-\alpha} \alpha\right\|<\frac{1}{2} \eta_{0} .
$$

This can be done with the aid of Journé's Lemma.

Fix a second small parameter $\eta_{\mathrm{J}}$ whose value will be specified below. (The subscript $\mathrm{J}$ is for 'Journé.') Apply Lemma 4.10. There is a set $V \supset \operatorname{sh}(\mathcal{U})$ and a function Emb $: \mathcal{U} \longrightarrow[1, \infty)$ for which these conditions hold.

- $|V|<1+\eta_{\mathrm{J}}$

- $\operatorname{Emb}(R) R \subset V$ for all $R \in \mathcal{U}$;

- $\|\widetilde{\alpha}\|_{\mathrm{BMO}\left(\mathbb{C}_{+}^{d}\right)} \leq K_{\eta_{\mathrm{J}}} \eta_{-1}$

where in the last line, we have

$$
\widetilde{\alpha} \stackrel{\text { def }}{=} \sum_{R \in \mathcal{U}} \operatorname{Emb}(R)^{-2 d}\left\langle b, v_{R}\right\rangle v_{R} .
$$

We now decompose the symbol $b$. We have already defined $\alpha$. Set

$$
\beta \stackrel{\text { def }}{=} \sum_{\substack{R \subset V \\ R \notin \mathcal{U}}}\left\langle b, v_{R}\right\rangle v_{R} .
$$


Thus, these are the rectangles with are 'close' to $\mathcal{U}$, but not in it, as defined by the set $V$. Define $\gamma$ by $b=\alpha+\beta+\gamma$. To verify (7.4), it suffices to show that

$$
\begin{aligned}
& \left\|\mathrm{H}_{\beta} \alpha\right\|<K \eta_{\mathrm{J}}^{1 / 4}, \\
& \left\|\mathrm{H}_{\gamma} \alpha\right\|<K_{\eta_{\mathrm{J}}} \eta_{-1} .
\end{aligned}
$$

One then specifies $\eta_{\mathrm{J}}$ so that the top line is no more than $\frac{1}{4} \eta_{0}$. The constant $K_{\eta_{\mathrm{J}}}$ that appears in the second line is absolute, so we can then fix $\eta_{-1}$ sufficiently small to prove (7.4).

The inequality for $\beta$ is easily available to us, by the particular form of the Journé Lemma we are using. Observe first that

$$
1+\sum_{\substack{R \subset V \\ R \notin \mathcal{U}}}\left|\left\langle b, v_{R}\right\rangle\right|^{2}=\sum_{R \subset V}\left|\left\langle b, v_{R}\right\rangle\right|^{2} \leq 1+\eta_{\mathrm{J}} .
$$

Therefore, $\|\beta\|_{2} \leq \sqrt{\eta_{\mathrm{J}}}$. On the other hand, the $\operatorname{BMO}\left(\mathbb{C}_{+}^{d}\right)$ norm of $\beta$ is less than or equal to one. Thus, we have $\|\beta\|_{4} \lesssim \eta^{1 / 4}$. A Hankel operator is at worst a product, thus

$$
\left\|\mathrm{H}_{\beta} \alpha\right\| \leq\|\beta\|_{4}\|\alpha\|_{4} \leq K \eta_{\mathrm{J}}^{1 / 4}
$$

So it remains to verify $(7.8)$.

An Initial Calculation. We make an explicit computation of a Hankel operator, in a manner similar to (3.9). Namely, restricting attention to one dimension, we have

$$
\mathrm{H}_{v_{I}} \overline{v_{J}}=\mathrm{P}_{+}\left(v_{I} \overline{v_{J}}\right)= \begin{cases}0 & 8|J|<|I| \\ \mathrm{P}_{+}\left(v_{I} \overline{v_{J}}\right) & |I| \leq 8|J| \leq 64|I| \\ v_{I} \overline{v_{J}} & |I|<8|J| .\end{cases}
$$

This follows from the Fourier localization properties of the Meyer wavelet. The Fourier support of the product $v_{I} \overline{v_{J}}$ is given by the convolution of the Fourier supports, which are specified by (2.10). If $J$ is much smaller than $I$, the product is purely antianalytic, giving us the first case above. In the third case, $v_{I} \overline{v_{J}}$ is purely analytic.

We apply the observation above to the term $\mathrm{H}_{\gamma} \bar{\alpha}$. This leads us to the conclusion that

$$
\begin{gathered}
\left\|\mathrm{H}_{\gamma} \bar{\alpha}\right\|=\left\|\sum_{\left(R, R^{\prime}\right) \in \mathcal{A}}\left\langle b, u_{R}\right\rangle \overline{\left\langle\varphi, u_{R}\right\rangle} u_{R} \overline{u_{R}},\right\|_{2} \\
\mathcal{A} \stackrel{\text { def }}{=}\left\{\left(R, R^{\prime}\right): R \subset U, R^{\prime} \not \subset V,\left|R_{s}^{\prime}\right| \leq 64\left|R_{s}\right|, 1 \leq s \leq d\right\} .
\end{gathered}
$$

It is essential to observe that this last sum can be written as a finite sum of the paraproducts in Theorem 5.4, applied to the functions $\alpha$ and $\gamma$. This sum varies of choices of $\vec{k}$ with $|\vec{k}| \leq 6$, and arbitrary $J \subset\{1, \ldots, d\}$. (The subset $J$ consists of those coordinates $s$ for which $\left|R_{s}\right|=2^{k_{s}}\left|R_{s}^{\prime}\right|$.) 
We use Theorem 5.4 to provide an estimate of the $L^{2}$ norm of the sum above an absolute constant times $\eta_{-1}$. In particular, we want to use the more technical estimate (5.7) to achieve this end.

We will need to decompose the collection $\mathcal{A}$ into appropriate parts to which this estimate applies. That is the purpose of this definition. For an integer $n \geq 1$, take

$$
\alpha_{n} \stackrel{\text { def }}{=} \sum_{\substack{R \subset U \\ 2^{n-1} \leq \operatorname{Enl}(R ; U) \leq 2^{n}}}\left\langle b, u_{R}\right\rangle u_{R}
$$

We claim that

$$
\left\|\mathrm{H}_{\gamma} \alpha_{n}\right\| \lesssim 2^{-n} \eta_{-1}
$$

It follows from Lemma 4.10 that we have the estimate

$$
\left\|\alpha_{n}\right\|_{\mathrm{BMO}\left(\mathbb{C}_{+}^{d}\right)} \lesssim 2^{2 d n} \eta_{-1},
$$

indeed, this is the point of this definition. From other parts of the expansion of the Hankel operator, we need to find some decay in $n$.

Nevertheless, from this estimate and the upper bound on Hankel operator norms, we have the estimate

$$
\left\|\mathrm{H}_{\gamma} \alpha_{n}\right\| \lesssim\|b\|_{\mathrm{BMO}\left(\mathbb{C}_{+}^{d}\right)}\left\|\alpha_{n}\right\|_{2} \lesssim 2^{2 d n} \eta_{-1} .
$$

We use this estimate for $n<20$, say.

For $n \geq 20, R \in \mathcal{U}$ with $2^{n-1} \leq \operatorname{Enl}(R ; \mathcal{U}) \leq 2^{n}$, and rectangle $R^{\prime}$ with $\left(R, R^{\prime}\right) \in \mathcal{A}$, it follows that we must have $2^{n-9} R \cap R^{\prime}=\emptyset$. That is, (5.6) is satisfied with the value of $A$ in that display being $A \simeq 2^{n}$ for $n \geq 20$. Thus, we conclude that

$$
\left\|\mathrm{H}_{\gamma} \alpha_{n}\right\| \lesssim 2^{-50 n} \eta_{-1}, \quad n \geq 20
$$

This completes our proof of (7.10), and the proof of the lower bound on the norm of Hankel operators.

\section{REFERENCES}

[1] V. M. Adamjan, D. Z. Arov, and M. G. Krĕ̌n, Analytic properties of the Schmidt pairs of a Hankel operator and the generalized Schur-Takagi problem, Mat. Sb. (N.S.) 86(128) (1971), 34-75. MR0298453 (45 \#7505) (Russian) $\uparrow 12$

[2] J. Bourgain, Some remarks on Banach spaces in which martingale difference sequences are unconditional, Ark. Mat. 21 (1983), 163-168. MR727340 (85a:46011) $\uparrow 6$ 
[3] D. L. Burkholder, A geometric condition that implies the existence of certain singular integrals of Banach-space-valued functions, Conference on Harmonic Analysis in Honor of Antoni Zygmund, Vol. I, II (Chicago, Ill., 1981), Wadsworth Math. Ser., Wadsworth, Belmont, CA, 1983, pp. 270-286. MR730072 (85i:42020) $\uparrow 6$

[4] Carlos Cabrelli, Michael Lacey, Ursula Molter, and Jill C Pipher, Variations on the Theme of Journe's Lemma, To appear in Houston J Math., arXiv:math.CA/0412174. $\uparrow 22$

[5] L. Carleson, A counterexample for measures bounded on $H^{p}$ spaces for the bidisk, Mittag-Leffler Rep. No. 7, Inst. Mittag-Leffler (1974). $\uparrow 20$

[6] Sun-Yung A. Chang and Robert Fefferman, Some recent developments in Fourier analysis and $H^{p}$-theory on product domains, Bull. Amer. Math. Soc. (N.S.) 12 (1985), 1-43. MR 86g:42038 ^2, 17

[7] - A continuous version of duality of $H^{1}$ with BMO on the bidisc, Ann. of Math. (2) 112 (1980), 179-201. MR 82a:32009 $\uparrow 17,20$

[8] Sun-Yung A. Chang, Carleson measure on the bi-disc, Ann. of Math. (2) 109 (1979), 613-620. $\uparrow$

[9] Sun-Yung A. Chang and Robert Fefferman, Some recent developments in Fourier analysis and $H^{p}$-theory on product domains, Bull. Amer. Math. Soc. (N.S.) 12 (1985), 1-43. MR 86g:42038 $\uparrow$

[10] - A continuous version of duality of $H^{1}$ with BMO on the bidisc, Ann. of Math. (2) 112 (1980), 179-201. MR 82a:32009 $\uparrow 2$

[11] S. Chanillo, A note on commutators, Indiana Univ. Math. J. 31 (1982), 7-16. MR 84j:42027 个14

[12] R. R. Coifman, R. Rochberg, and Guido Weiss, Factorization theorems for Hardy spaces in several variables, Ann. of Math. (2) 103 (1976), 611-635. MR 54 \#843 个14

[13] Mischa Cotlar and Cora Sadosky, The Helson-Szegö theorem in $L^{p}$ of the bidimensional torus, Harmonic Analysis and Partial Differential Equations (Boca Raton, FL, 1988), Contemp. Math., vol. 107, Amer. Math. Soc., Providence, RI, 1990, pp. 19-37. MR1066468 (92a:42021) $\uparrow 13$

[14] _ Two-parameter lifting theorems and double Hilbert transforms in commutative and noncommutative settings, J. Math. Anal. Appl. 150 (1990), 439-480. MR1067439 (91f:47006) 个

[15] _ . Transference of metrics induced by unitary couplings, a Sarason theorem for the bidimensional torus, and a Sz.-Nagy-Foias theorem for two pairs of dilations, J. Funct. Anal. 111 (1993), 473-488. MR1203463 (94g:47007) $\uparrow$

[16] _ Abstract, weighted, and multidimensional Adamjan-Arov-Krein theorems, and the singular numbers of Sarason commutants, Integral Equations Operator Theory 17 (1993), 169-201. MR1233667 (94k:47040) $\uparrow 13$

[17] - Nehari and Nevanlinna-Pick problems and holomorphic extensions in the polydisk in terms of restricted BMO, J. Funct. Anal. 124 (1994), 205-210. MR1284610 (95f:47047) 225

[18] — The Adamjan-Arov-Krein theorem in general and regular representations of $\mathbf{R}^{2}$ and the symplectic plane, Toeplitz Operators and Related Topics (Santa Cruz, CA, 1992), Oper. Theory Adv. Appl., vol. 71, Birkhäuser, Basel, 1994, pp. 54-78. MR1300214 (95h:47032) $\uparrow 13$

[19] _ Two distinguished subspaces of product BMO and Nehari-AAK theory for Hankel operators on the torus, Integral Equations Operator Theory 26 (1996), 273-304. MR1415032 (98e:47043) $\uparrow 13$

[20] _ _ A polydisk version of Beurling's characterization for invariant subspaces of finite multicodimension, Operator Theory for Complex and Hypercomplex Analysis (Mexico City, 1994), Contemp. Math., vol. 212, Amer. Math. Soc., Providence, RI, 1998, pp. 51-56. MR1486589 (98k:30047) $\uparrow$

[21] D. Cruz-Uribe and A. Fiorenza, Endpoint estimates and weighted norm inequalities for commutators of fractional integrals, Publ. Mat. 47 (2003), 103-131. MR 2004a:42010 114

[22] C. Fefferman and E. M. Stein, $H^{p}$ spaces of several variables, Acta Math. 129 (1972), 137-193. MR 56 $\# 6263 \uparrow 12$

[23] Robert Fefferman, Harmonic analysis on product spaces, Ann. of Math. (2) 126 (1987), 109-130. MR 90e: $42030 \uparrow 14,17$ 
[24] R. Fefferman, A note on Carleson measures in product spaces, Proc. Amer. Math. Soc. 93 (1985), 509511. MR 86f:32004 个

[25] _ Bounded mean oscillation on the polydisk, Ann. of Math. (2) 110 (1979), 395-406. MR 81c:32016 $\uparrow 17,20$

[26] Robert Fefferman and Elias M. Stein, Singular integrals on product spaces, Adv. in Math. 45 (1982), 117-143. MR664621 (84d:42023) 个

[27] Sarah H. Ferguson and Michael T. Lacey, A characterization of product BMO by commutators, Acta Math. 189 (2002), 143-160. $1961195 \uparrow 1,2,22,25$

[28] Sarah H. Ferguson and Cora Sadosky, Characterizations of bounded mean oscillation on the polydisk in terms of Hankel operators and Carleson measures, J. Anal. Math. 81 (2000), 239-267. MR 2001h:47040 $\uparrow$

[29] M. Seetharama Gowda, Nonfactorization theorems in weighted Bergman and Hardy spaces on the unit ball of $\mathbf{C}^{n}(n \geq 1)$, Trans. Amer. Math. Soc. 277 (1983), 203-212. MR690048 (84i:32005) $\uparrow 7$

[30] Caixing Gu and Dechao Zheng, The semi-commutator of Toeplitz operators on the bidisc, J. Operator Theory 38 (1997), 173-193. MR1462020 (98g:47022) $\uparrow$

[31] Charles Horowitz, Zeros of functions in the Bergman spaces, Bull. Amer. Math. Soc. 80 (1974), 713-714. MR0338399 (49 \#3164) $\uparrow 7$

[32] _ Factorization theorems for functions in the Bergman spaces, Duke Math. J. 44 (1977), 201-213. MR0427650 (55 \#681) $\uparrow 7$

[33] Svante Janson, Mean oscillation and commutators of singular integral operators, Ark. Mat. 16 (1978), 263-270. MR 80j:42034 个

[34] Peter W. Jones and Jean-Lin Journé, On weak convergence in $H^{1}\left(\mathbf{R}^{d}\right)$, Proc. Amer. Math. Soc. 120 (1994), 137-138. MR1159172 (94b:42011) $\uparrow$

[35] Jean-Lin Journé, A covering lemma for product spaces, Proc. Amer. Math. Soc. 96 (1986), 593-598. MR $87 \mathrm{~g}: 42028 \uparrow 2,17,21$

[36] _ Calderón-Zygmund operators on product spaces, Rev. Mat. Iberoamericana 1 (1985), 55-91. MR 88d:42028 个2, 17, 23

[37] Jason Metcalfe and Michael T. Lacey, Paraproducts in One and Several Parameters, arXiv:math.CA/0502334. $\uparrow 23$

[38] Michael T. Lacey and Erin Terwelleger, Little Hankel Operators and Product BMO (2004). ^1, 2, 22, 25

[39] Brett Wick, Michael T. Lacey, and Erin Terwelleger, Remarks on Product VMO, Proceedings AMS, Posted July 7, 2005, (to appear in print), arXiv:math.CA/0405097. $\uparrow$

[40] Michael $\mathrm{T}$ Lacey, Commutators with Reisz Potentials in One and Several Parameters, arXiv:math.CA/0502336. $\uparrow 14,15,17$

[41] Kent G. Merryfield, On the area integral, Carleson measures and $H^{p}$ in the polydisc, Indiana Univ. Math. J. 34 (1985), 663-685. MR794581 (87c:42023) 个

[42] Yves Meyer and Ronald Coifman, Wavelets, Cambridge Studies in Advanced Mathematics, vol. 48, Cambridge University Press, Cambridge, 1997, ISBN 0-521-42001-6, 0-521-79473-0, Calderón-Zygmund and multilinear operators; Translated from the 1990 and 1991 French originals by David Salinger. MR $98 \mathrm{e}: 42001 \uparrow 6$

[43] Joseph Miles, A factorization theorem in $H^{1}\left(U^{3}\right)$, Proc. Amer. Math. Soc. 52 (1975), 319-322. MR0374459 (51 \#10659) $\uparrow 7$

[44] Camil Muscalu, Jill Pipher, Terrance Tao, and Christoph Thiele, Bi-parameter paraproducts, arxiv:math.CA/0310367. $\uparrow 23$

[45] _ Multi-parameter paraproducts, arXiv:math.CA/0411607. $\uparrow 23$

[46] Fedor Nazarov, Serguei Treil, and Alexander Volberg, Counterexample to the infinite-dimensional Carleson embedding theorem, C. R. Acad. Sci. Paris Sér. I Math. 325 (1997), 383-388. MR 98d:46039 (English, with English and French summaries) $\uparrow 6$ 
[47] F. Nazarov, G. Pisier, S. Treil, and A. Volberg, Sharp estimates in vector Carleson imbedding theorem and for vector paraproducts, J. Reine Angew. Math. 542 (2002), 147-171. MR 2002m:47038 $\uparrow 6$

[48] F. L. Nazarov and S. R. Treel', The hunt for a Bellman function: applications to estimates for singular integral operators and to other classical problems of harmonic analysis, Algebra i Analiz 8 (1996), 32162. MR1428988 (99d:42026) (Russian, with Russian summary) $\uparrow 6$

[49] F. Nazarov, S. Treil, and A. Volberg, Cauchy integral and Calderón-Zygmund operators on nonhomogeneous spaces, Internat. Math. Res. Notices (1997), 703-726. MR1470373 (99e:42028) 66

[50] _ Weak type estimates and Cotlar inequalities for Calderón-Zygmund operators on nonhomogeneous spaces, Internat. Math. Res. Notices (1998), 463-487. MR1626935 (99f:42035) $\uparrow 6$

[51] F. Nazarov, G. Pisier, S. Treil, and A. Volberg, Sharp estimates in vector Carleson imbedding theorem and for vector paraproducts, J. Reine Angew. Math. 542 (2002), 147-171. MR1880830 (2002m:47038) $\uparrow 6$

[52] F. Nazarov and A. Volberg, The Bellman function, the two-weight Hilbert transform, and embeddings of the model spaces $K_{\theta}$, J. Anal. Math. 87 (2002), 385-414. MR1945290 (2003j:30081) $\uparrow 6$

[53] Z. Nehari, On bounded bilinear forms, Ann. of Math. (2) 65 (1957), 153-162. $\uparrow 11$

[54] Nikolai K. Nikolski, Operators, functions, and systems: an easy reading. Vol. 1, Mathematical Surveys and Monographs, vol. 92, American Mathematical Society, Providence, RI, 2002, ISBN 0-8218-1083-9. MR1864396 (2003i:47001a) 113

[55] _ Operators, functions, and systems: an easy reading. Vol. 2, Mathematical Surveys and Monographs, vol. 93, American Mathematical Society, Providence, RI, 2002, ISBN 0-8218-2876-2. MR1892647 (2003i:47001b) $\uparrow 13$

[56] M. Paluszyński, Characterization of the Besov spaces via the commutator operator of Coifman, Rochberg and Weiss, Indiana Univ. Math. J. 44 (1995), 1-17. MR 96j:42006 个

[57] Vladimir V. Peller, Hankel operators and their applications, Springer Monographs in Mathematics, Springer-Verlag, New York, 2003, ISBN 0-387-95548-8. MR1949210 (2004e:47040) $\uparrow 13$

[58] Stefanie Petermichl, Dyadic shifts and a logarithmic estimate for Hankel operators with matrix symbol, C. R. Acad. Sci. Paris Sér. I Math. 330 (2000), 455-460. MR 2000m:42016 (English, with English and French summaries) $\uparrow 6,15$

[59] S. Petermichl, S. Treil, and A. Volberg, Why the Riesz transforms are averages of the dyadic shifts?, Proceedings of the 6th International Conference on Harmonic Analysis and Partial Differential Equations (El Escorial, 2000), 2002, pp. 209-228. MR1964822 (2003m:42028) 个6, 15

[60] Jill Pipher, Journé's covering lemma and its extension to higher dimensions, Duke Math. J. 53 (1986), 683-690. MR 88a:42019 $\uparrow 22$

[61] Sandra Pott and Martin P. Smith, Paraproducts and Hankel operators of Schatten class via p-JohnNirenberg theorem, J. Funct. Anal. 217 (2004), 38-78. MR2097606 $\uparrow$

[62] Sandra Pott and Cora Sadosky, Bounded mean oscillation on the bidisk and operator BMO, J. Funct. Anal. 189 (2002), 475-495. MR1892177 (2004b:47056) 个

[63] Jean-Pierre Rosay, Sur la non-factorisation des éléments de l'espace de Hardy $H^{1}\left(U^{2}\right)$, Illinois J. Math. 19 (1975), 479-482. MR0377098 (51 \#13272) (French) $\uparrow 7$

[64] Walter Rudin, Function theory in polydiscs, W. A. Benjamin, Inc., New York-Amsterdam, 1969. MR0255841 (41 \#501) $\uparrow 7$

[65] Elias M. Stein, Some geometrical concepts arising in harmonic analysis, Geom. Funct. Anal. (2000), 434-453. MR1826263 (2002f:42014) 个

[66] Ferenc Weisz, $(C, \alpha)$ means of several-parameter Walsh- and trigonometric-Fourier series, East J. Approx. 6 (2000), 129-156. MR1767858 (2001k:42015) ^14

E-mail address: lacey@math.gatech.edu 